\title{
A radiative transfer module for calculating photolysis rates and solar heating in climate models: Solar-J v7.5
}

\author{
Juno Hsu ${ }^{1}$, Michael J. Prather ${ }^{1}$, Philip Cameron-Smith ${ }^{2}$, Alex Veidenbaum ${ }^{3}$, and Alex Nicolau ${ }^{3}$ \\ ${ }^{1}$ Department of Earth System Science, University of California Irvine, Irvine, California, 92697, USA \\ ${ }^{2}$ Lawrence Livermore National Laboratory, Livermore, California, 94551, USA \\ ${ }^{3}$ Department of Computer Science, University of California Irvine, Irvine, California, 92697, USA
}

Correspondence to: Juno Hsu (junoh@uci.edu)

Received: 31 January 2017 - Discussion started: 8 February 2017

Revised: 25 May 2017 - Accepted: 30 May 2017 - Published: 3 July 2017

\begin{abstract}
Solar-J is a comprehensive radiative transfer model for the solar spectrum that addresses the needs of both solar heating and photochemistry in Earth system models. Solar-J is a spectral extension of Cloud-J, a standard in many chemical models that calculates photolysis rates in the $0.18-0.8 \mu \mathrm{m}$ region. The Cloud-J core consists of an eightstream scattering, plane-parallel radiative transfer solver with corrections for sphericity. Cloud-J uses cloud quadrature to accurately average over correlated cloud layers. It uses the scattering phase function of aerosols and clouds expanded to eighth order and thus avoids isotropic-equivalent approximations prevalent in most solar heating codes. The spectral extension from 0.8 to $12 \mu \mathrm{m}$ enables calculation of both scattered and absorbed sunlight and thus aerosol direct radiative effects and heating rates throughout the Earth's atmosphere.

The Solar-J extension adopts the correlated-k gas absorption bins, primarily water vapor, from the shortwave Rapid Radiative Transfer Model for general circulation model (GCM) applications (RRTMG-SW). Solar-J successfully matches RRTMG-SW's tropospheric heating profile in a clear-sky, aerosol-free, tropical atmosphere. We compare both codes in cloudy atmospheres with a liquid-water stratus cloud and an ice-crystal cirrus cloud. For the stratus cloud, both models use the same physical properties, and we find a systematic low bias of about $3 \%$ in planetary albedo across all solar zenith angles caused by RRTMG-SW's two-stream scattering. Discrepancies with the cirrus cloud using any of RRTMG-SW's three different parameterizations are as large as about $20-40 \%$ depending on the solar zenith angles and occur throughout the atmosphere.
\end{abstract}

Effectively, Solar-J has combined the best components of RRTMG-SW and Cloud-J to build a high-fidelity module for the scattering and absorption of sunlight in the Earth's atmosphere, for which the three major components - wavelength integration, scattering, and averaging over cloud fields - all have comparably small errors. More accurate solutions with Solar-J come with increased computational costs, about 5 times that of RRTMG-SW for a single atmosphere. There are options for reduced costs or computational acceleration that would bring costs down while maintaining improved fidelity and balanced errors.

\section{Introduction}

A major challenge in simulating the Earth's climate is the tracking of solar energy, its absorption, and scattering within and reflection from the Earth system in the presence of heterogeneously distributed clouds and aerosols. The fifth assessment of Intergovernmental Panel on Climate Change (IPCC, chap. 7, Boucher et al., 2013) summarizes that the net radiative feedback due to all cloud types is likely to be positive but with large uncertainty, mostly attributed to the uncertain impact of warming on low clouds. The confidence in the aerosol-climate feedback, through both aerosol and cloud albedo, is even lower, and the uncertainty is $\pm 0.2 \mathrm{~W} \mathrm{~m}^{-2}{ }^{\circ} \mathrm{C}^{-1}$. Major modeling challenges naturally point to the sub-grid parameterizations of clouds and cloud-aerosol interactions in coarsely gridded global models, and the IPCC reports have documented substantial developments in the modeling of the chemical-physical properties 
of aerosols and clouds (Boucher et al., 2013). In comparison, relatively little attention has been paid to improving the treatment of aerosol and cloud scattering in climate models. This is both surprising and not. Solutions of the radiative transfer (RT) equations in scattering media are well documented with numerous methods and readily available packages such as TUV (Tie et al., 2003; Palancar et al., 2011) and SCIATRAN (Rozanov et al., 2014). However, these more accurate reference codes have always been viewed as too computationally expensive. In terms of climate model development, this is a solved problem with little intellectual interest but is too onerous to improve, and thus low-order approximations remain in place.

We present here Solar-J version 7.5, a radiative transfer model based on the computationally optimized photolysis code, Cloud-J (Prather, 2015). Cloud-J combined an improved cloud overlap algorithm based on observed decorrelation lengths with the cloud quadrature scheme (Neu et al., 2007) for averaging over independent column atmospheres. It includes an eight-stream scattering photolysis code with semi-spherical geometry and wavelength integration over 18 spectral bins from 177 to $850 \mathrm{~nm}$. In the most recent version (v7.4), the spectrum was shortened to $778 \mathrm{~nm}$ (see Fig. 1). Prior released versions, known in the community as FastJX or simply Fast-J (see Søvde et al., 2012; Telford et al., 2013; Sukhodolov et al., 2016), were based on the work of Fast-J2 (Bian and Prather, 2002) and the original Fast-J (Wild et al., 2000), separately optimizing the absorptions of photon fluxes into 12 stratospheric bins (177-292 nm) and seven tropospheric bins $(292-850 \mathrm{~nm})$. Although this is the first version of Solar-J, we retain the numbering of the released versions of the core photolysis code. For simplicity, we will refer to Cloud-J, not Fast-J, as the starting point of Solar-J throughout the text. The accurate treatment of cloud and aerosol scattering has been an essential requirement for atmospheric chemistry modeling, and Cloud-J or alternative models (fast-TUV, Tie et al., 2003) are used standardly in global chemistry models. Solar-J is an extension of the Cloud-J wavelength range $(0.18-0.8 \mu \mathrm{m})$ out to $12 \mu \mathrm{m}$ and includes an eight-stream scattering solution for the absorption and reflection of sunlight over the full spectrum. Scattering and absorption by large aerosols (dust) and clouds are important for heating rates at these longer wavelengths. The longterm goal is to develop Solar-J as a single module for climate models, albeit with increased computational cost, delivering photolysis rates and more accurate shortwave heating rates, particularly for aerosol and cloud radiative forcing.

As finer grid resolutions and massively parallel computing are being pursued to enable more realistic atmospheric interactions with the land, ocean, and biosphere in climate modeling, the radiative transfer codes implemented in most of the global models remain in their simplest possible analytical form of two-stream scattering. With this approximation, all upward and downward scattering occurs at a single angle, and the scattering must be treated as isotropic, i.e., indepen- dent of sun angle. The ubiquitous adoption of two-stream RT codes by the global climate and weather-forecasting models (e.g., the US Department of Energy (DOE) Accelerated Climate Modeling for Energy (ACME), the National Center for Atmospheric Research (NCAR) Community Earth System Model (CESM), the European Centre for MediumRange Weather Forecasts (ECMWF) model) has been enabled by standardized packages like the Rapid Radiative Transfer Model for general circulation model (GCM) applications (RRTMG), developed based on the correlated-k approach (Mlawer et al., 1997; Clough et al., 2005). A twostream model was certainly necessary at a time when the need for computational efficiency exceeded that for accuracy. With the rapid advancement of massively parallel computing, it is time to ask if an upgrade to a higher-order scheme is needed for improved accuracy in climate modeling, particularly with regard to cloud and aerosol forcing. The twostream scattering approximation has been in use for decades in climate models and evaluating its systematic errors remains an active research topic ( $\mathrm{Li}$ et al., 2015; Barker et al., 2015). The errors are mostly from the inadequacy of using a single angle to represent the scattering of cloud particles and aerosols. For example, the anisotropic, forward-peaked scattering of all relevant atmospheric aerosols and cloud particles cannot be represented with the two-stream approach, and all scattering must be reduced to isotropic. To address this problem, a commonly used delta-scaling technique is applied by removing the large forward-scattering peak, thus reducing the optical depth (Joseph et al., 1976; Wiscombe, 1977). In addition, the Henyey-Greenstein (HG) phase function (Henyey and Greenstein, 1941) is often used to tune the two-stream scattering to better represent the scattering of large particles for specific sun angles. Unfortunately, the HG phase function lacks the realistic back-scattering peak found for cloud particles, particularly ice crystals (Zhou and Yang, 2015). Li et al. (2015) find biases caused by the HG phase function and conclude that higher-order moments of the phase function coupled with a multi-stream radiative transfer algorithms are needed to improve accuracy. They demonstrate this point with a four-stream delta-Eddington code developed by Li and Ramaswamy (1996). Wild et al. (2000) tested the accuracy of different-order codes for computing the mean radiation field in the presence of thick water clouds and found that eight streams were necessary to have errors of only a few percent relative to a 160 -stream code that resolved the scattering phase function. For Solar-J, we adopt the Wild et al. (2000) optimization for water clouds and use Mie (liquid) or Mishchenko (ice) (Mishchenko et al., 1996, 2004) full phase functions for scattering, truncate the expansion in Legendre polynomials to order 8, and solve the scattering with eight streams with no delta scaling of the optical depth.

The Solar-J model and tests are described in Sect. 2. The resulting comparisons with RRTMG-SW are presented in Sect. 3. In this paper, we use RRTMG as shorthand for 
Solar-J spectrum: merging Fast-J/Cloud-J with RRTMG-SW

\begin{tabular}{|c|c|c|c|c|}
\hline $\begin{array}{l}\text { Fast-J } \\
\text { (Wild et. al, 2000) } \\
\text { (Bian \& Prather, 2002) }\end{array}$ & $\begin{array}{l}\text { Bins } 1-17 \\
(177-412 \mathrm{~nm})\end{array}$ & \multicolumn{2}{|c|}{ Bin-18 (412-850 nm) } & \\
\hline $\begin{array}{l}\text { Cloud-J v7.4 } \\
\text { (Prather, 2015) }\end{array}$ & $\begin{array}{l}\text { Bins } 1-17 \\
(177-412 \mathrm{~nm})\end{array}$ & \multicolumn{2}{|c|}{ Bin-18 (412-778 nm) } & \\
\hline $\begin{array}{l}\text { Solar-J v7.5 } \\
\text { (S-bins) } \\
\text { (this study) }\end{array}$ & $\begin{array}{l}\text { Bins } 1-17 \\
(177-412 \mathrm{~nm})\end{array}$ & $\begin{array}{l}\text { Bin-18 }{ }^{\mathrm{a}} \\
(412-778 \mathrm{~nm}) \\
\text { Cloud-J's } \mathrm{O}_{3} \\
+ \text { weak } \mathrm{O}_{2}+\mathrm{H}_{2} \mathrm{O} \\
\text { (1 sub-bin) }\end{array}$ & $\begin{array}{l}\text { Bin- } 18^{b} \\
(442-778 \mathrm{~nm}) \\
\text { Surface } \mathrm{H}_{2} \mathrm{O}+\mathrm{O}_{2} \\
\text { (4 sub-bins) }\end{array}$ & $\begin{array}{l}\text { Bins } 19-27 \\
(778-12195 \mathrm{~nm}) \\
\text { (78 sub-bins) }\end{array}$ \\
\hline $\begin{array}{l}\text { RRTMG } \\
\text { (Mlawer et. al, 1997) }\end{array}$ & $\begin{array}{l}\text { Band no. } 26-28 \\
(200-442 \mathrm{~nm}) \\
\text { (20 sub-bins) }\end{array}$ & & $\begin{array}{l}\text { Bands } 24 \& 25 \\
(442-778 \mathrm{~nm}) \\
\text { (14 sub-bins) }\end{array}$ & $\begin{array}{l}\text { Bands } 16-23+ \\
\text { Band } 29 \\
\text { (778-12195 nm) } \\
\text { (78 sub-bins) }\end{array}$ \\
\hline
\end{tabular}

Figure 1. Solar-J extends Cloud-J's solar wavelength bands by combining and modifying RRTMG-SW's bands 24 and 25 (442-778 nm) and adopts all RRTMG's bands longwards of $778 \mathrm{~nm}$ (see text for details).

RRTMG-SW. Section 4 examines computational costs for Solar-J and options for optimization. Conclusions and a path forward are discussed in Sect. 5.

\section{Methods: model configuration and test cases}

\subsection{Solar-J spectral configuration}

The 18 bins of Cloud-J make up the first 18 bins of Solar$\mathrm{J}$ and were optimized for calculating photolysis rates below $64 \mathrm{~km}$ (Wild et al., 2000; Bian and Prather, 2002). The first 11 bins (177-291 nm) are optimized around the SchumannRunge bands of $\mathrm{O}_{2}$ and the Hartley bands of $\mathrm{O}_{3}$, and the next 7 bins are optimized for tropospheric photolysis (291$778 \mathrm{~nm}$ ). The bins were chosen to have relatively uniform opacities for the principal absorbing species $\mathrm{O}_{2}$ and $\mathrm{O}_{3}$ across the wavelengths in each bin. In some cases, this includes combining different wavelength regions on either side of the $\mathrm{O}_{3}$ maximum cross section near $255 \mathrm{~nm}$. Effectively, the 18 bins extend the use of opacity distribution functions used to calculate $\mathrm{O}_{2}$ photolysis rates in the Schumann-Runge bands (Fang et al., 1974), an equivalent to the correlated-k method in the infrared (Lacis and Oinas, 1991). An inherent assumption is that any other scatterers and absorbers are uniform across each wavelength bin, justified by the narrowness of the bins and the lack of sharp spectral features in clouds and aerosols. Because Cloud-J has been optimized against high-resolution spectral data for stratospheric ozone photolysis and continually updated with new cross sections (Sander et al., 2011), and tested against other codes (Palancar et al., 2011; PhotoComp, Eyring et al., 2010), we have confidence in our stratospheric photolysis and heating rates.
The large bin $18(412-778 \mathrm{~nm})$ that includes the $\mathrm{O}_{3}$ Chappuis band is unusual for Cloud-J: it assumes a uniform absorption cross section for $\mathrm{O}_{3}$, and it has a large, factor of 2 change in wavelength. The $\mathrm{O}_{3}$ cross sections vary smoothly over bin 18 and are $>0.5 \times 10^{-21} \mathrm{~cm}^{2}$ over the range $475-$ $725 \mathrm{~nm}$ with a broad maximum of $5 \times 10^{-21} \mathrm{~cm}^{2}$ about $600 \mathrm{~nm}$. Overhead opacity ranges from 0.4 to $4 \%$ over this band. With optically thin absorption, one can use the fluxweighted average cross section, $1.94 \times 10^{-21} \mathrm{~cm}^{2}$, for the entire bin. Both the attenuation of sunlight and the absorption of photons to calculate the $\mathrm{O}_{3}$ photolysis rate use this average. At very large air masses (solar zenith angles of $89-95^{\circ}$ ) the atmospheric path (vertical optical path $\times 1 / \cos (\mathrm{SZA})$ ) in the Chappuis band approaches 1 and modest errors appear. If highly accurate calculation of the photolysis and heating rates due in the Chappuis band is required, then further analysis of bin 18 is warranted, but otherwise this treatment is sufficiently accurate to follow these rates as the sun sets. Another possible source of error is that these cross sections are photon weighted, and for heating rates the cross sections should be energy weighted $\left(\mathrm{W} \mathrm{m}^{-2}\right)$. Fortunately, the energy-weighted $\mathrm{O}_{3}$ cross section, $1.91 \times 10^{-21} \mathrm{~cm}^{2}$, differs little from the photon-weighted one (with the result of $<0.04 \mathrm{~K} \mathrm{day}^{-1}$ difference in clear-sky stratospheric heating).

RRTMG-SW has nine large bins extending to wavelengths longer than the end point of Cloud-J, and we adopt the fluxweighted average optical properties of clouds and aerosols for these bins as an extension to Cloud-J v7.4 to become Solar-J v7.5. Figure 1 shows the overlap of the spectral bins of Fast-J v7.3, Solar-J v7.5, and RRTMG-SW. Also shown is the revised Cloud-J v7.4 for which the long-wavelength edge of bin 18 has been shortened from 850 to $778 \mathrm{~nm}$ to match 
the transition to RRTMG bins. The flux-weighted cross sections for several Cloud-J species have been recalculated to account for this. Be aware that these rescaled cross sections apply to Cloud-J versions 7.4 and later. Cloud-J remains a key component of Solar-J, as it produces representative samples of independent column atmospheres after considering the topology of cloud fractions (Prather, 2015). Solar-J has 27 major bins, referred to here as S-bins, e.g., S1-S27 in Table 4. Bins S1-S17 are taken directly from Cloud-J and have no sub-bins. The transition bin S18 combines CloudJ's uniform treatment of Chappuis-band $\mathrm{O}_{3}$ absorption with four small non-overlapping sub-bins (17.5 out of a total of $608.7 \mathrm{~W} \mathrm{~m}^{-2}$ ) to include RRTMG's $\mathrm{H}_{2} \mathrm{O}$ and $\mathrm{O}_{2}$ absorptions from their bins B24-B25. These four sub-bins have strong cross sections with their own distinct optical depth structures, and they do not overlap with the major $\mathrm{O}_{3}$ absorption in bin S18. The rest of the non-ozone sub-bins (weak cross sections) are lumped into one more sub-bin and added to Solar-J's Chappuis band. In all, we took RRTMG's 14 subbins from B24 and B25 and optimized these into 5 sub-bins for S18. The last 9 bins, S19-S27, are directly implemented from RRTMG and contain 78 sub-bins. The logic of having wavelength bins and then sub-bins within them is to allow the gaseous absorbers with similar opacities to be gathered into one sub-bin but to treat the scattering and absorption by aerosols and clouds as uniform across the major bin (see Sect. 2.2). The fidelity of the spectral extension of Solar-J to match RRTMG-SW is verified with the clear-sky case presented in Sect. 3.1.

\subsection{Clouds and aerosols}

Like the photolysis rates calculated in Cloud-J, the heating rates in RRTMG-SW and Solar-J are highly sensitive to the scattering and absorption from tropospheric and stratospheric aerosols, and from liquid-water and ice-water clouds. Cloud-J v7.4 has pre-computed tables of optical properties for typical aerosols and for both liquid- and ice-water clouds. For bins S1-S18, many of these are effectively nonabsorbing. With the extension to longer wavelengths, it becomes important to treat the absorption by clouds and the stratospheric sulfate layer. We take the refractive indices for liquid water, ice water, and sulfuric acid and calculate solarflux-weighted mean values for each bin (S12-S27). Sunlight over wavelengths for bins S1-S11 does not reach the troposphere in significant amounts, and hence the optical properties of bin S12 are used for S1-S11 for simplicity. For the first 18 bins, optical properties are weighted by the solar photon flux (photons $\mathrm{cm}^{-2} \mathrm{~s}^{-1}$ ), and for the last 9 bins they are weighted by the solar energy flux $\left(\mathrm{W} \mathrm{m}^{-2}\right)$. These refractive indices are combined with a Mie scattering code and a model for the size distribution of particles to calculate the effective radius $\left(r_{\mathrm{e}}\right.$ ), single scattering albedo (SSA), ratio of optical to geometric cross section $(Q)$, and the first eight terms in the expansion of the scattering phase function $\left(A_{0: 7}\right)$ that include the asymmetry parameter $\left(g=A_{1} / 3\right)$.

For liquid water, we take the refractive index from FORTRAN codes developed at the University of WisconsinMadison by M. A. Walters for liquid water (NDXWATER: Hale and Querry, 1973; Palmer and Williams, 1974; Downing and Williams, 1975) and ice water (NDXICE, based on Warren, 1984). Liquid-water clouds use Deirmendjian's C.1 gamma distribution of drop sizes $(\alpha=6$; see Deirmendjian, $1969)$ and the Mie code from Hansen and Travis (1974) for a range of effective radii: $r_{\mathrm{e}}=1.5,3,6,12,24,48 \mu \mathrm{m}$; see also Hess et al. (1998). Optical properties (SSA, $Q, A_{0: 7}, g$ ) are calculated for bins S12-S27 for these effective radii and then individual cloud properties at each bin are interpolated piecewise linearly in $r_{\mathrm{e}}$.

For ice-water clouds, we have two T-matrix computations supplied by M. Mishchenko for Cloud-J (Mishchenko et al., 2004) for warm (irregular) and cold (hexagonal) ice clouds. These included $Q$ and the scattering phase function (including $\left.A_{0: 7}\right)$ for the visible region $(\sim 600 \mathrm{~nm})$ and were used at all Solar-J wavelengths. When there is significant absorption, the values of SSA, and to some extent $Q$, are complex functions of $r_{\mathrm{e}}$ and do not simply scale as total mass. For this first version of Solar-J, we made a simplifying assumption and used the Mie code with the ice-water refractive index to calculate SSA and $Q$ as a function of $r_{\mathrm{e}}=3,6,12,24,48$, $96 \mu \mathrm{m}$ using the liquid-water clouds' C. 1 distribution. Effectively, we assumed that the ice particles were spheres. For the phase function $A_{0: 7}$, we kept the two T-matrix results (irregular and hexagonal ice particles) and used them for all $r_{\mathrm{e}}$ of that type of ice cloud. The obvious next upgrade to Solar-J is a redo of the ice-water clouds with a broader, better mix of cloud types (Mishchenko et al., 2016; Yang et al., 2015).

The refractive index for mixtures of sulfuric acid and water is also well characterized (Beyer et al., 1996; Biermann et al., 2000; Krieger et al., 2000; Lund-Myhre et al., 2003), and we use the tables from Lund-Myhre et al. (2003). For the stratospheric sulfate layer, we chose background and volcanic bimodal log-normal size distributions based on Deshler et al. (2003): the background distribution has a dominant mode $(98 \%)$ with $r_{\mathrm{e}}=0.125 \mu \mathrm{m}$ and a secondary mode with $r_{\mathrm{e}}=0.432 \mu \mathrm{m}$ for an average of $r_{\mathrm{e}}=0.131 \mu \mathrm{m}$; the volcanic distribution has a dominant mode $(81 \%)$ with $r_{\mathrm{e}}=0.487 \mu \mathrm{m}$ and a secondary mode with $r_{\mathrm{e}}=0.149 \mu \mathrm{m}$ for an average of $r_{\mathrm{e}}=0.422 \mu \mathrm{m}$. The stratospheric aerosol properties are tabulated for bins S5-S27 for a combination of temperatures (220-250-280 K) and weight-percent sulfuric acid (50-70$90 \%$ ) with $220 \mathrm{~K}$ and $70 \%$ being typical for the stratosphere (McGouldrick et al., 2011). The refractive indices and size distributions of tropospheric aerosols are not as well characterized. Cloud-J has a collection of aerosol optical properties for wavelengths $300-800 \mathrm{~nm}$ based on community contributions (e.g., Liousse et al., 1996; Martin et al., 2003), and this has been propagated for testing in Solar-J. However, if heating by tropospheric aerosols such as brown and black carbon 
and dust is to be accurately modeled with Solar-J, then one must go to the specific models to acquire the physical and optical properties, e.g., NCAR's CESM 1.2 (Tilmes et al., 2015).

The Solar-J bins, solar fluxes $\left(S_{\text {phot }}\right.$ in photons $\mathrm{cm}^{-2} \mathrm{~s}^{-1}$ and $S_{\text {Watt }}$ in $\mathrm{W} \mathrm{m}^{-2}$ ), and Rayleigh cross sections $\left(X_{\text {Rayl }} \mathrm{cm}^{2}\right)$ are summarized in Table 1 . The spectral properties for examples of liquid-water clouds $\left(r_{\mathrm{e}}=12 \mu \mathrm{m}\right)$, ice-water clouds ( $r_{\mathrm{e}}=48 \mu \mathrm{m}$, cold, hexagonal), background stratospheric $70 \mathrm{wt} \%$ sulfuric acid aerosols, and volcanically enhanced stratospheric aerosols for each Solar-J bin are given in Table 2. This table gives wavelength data for the real and imaginary refractive indices based on the flux-weighted means, as well as the Mie-derived values for $Q$, SSA, and $g$. The relative importance of cloud heating in each bin can be estimated by multiplying the solar energy by the absorbing fraction, $S_{\text {Watt }} \times(1-$ SSA). One finds that absorption for bins S1-S20 is negligible, that both types of clouds and stratospheric sulfate aerosols have large absorption in bins S25S27, and that ice-water clouds have large absorption per optical depth in bins S21-S24 while liquid-water clouds do not. Ice-water and liquid-water clouds have real refractive indices that differ by at most $5 \%$, and imaginary refractive indices that differ typically by a factor of 2 (except for S27). The cause of this difference in specific absorption is the ratio of mass (which controls absorption) to surface area (which controls optical depth), i.e., it is proportional to $r_{\mathrm{e}}$, and ice-water clouds typically have 4 times greater $r_{\mathrm{e}}$.

\subsection{Test cases: clear-sky, clouds, and the optical properties}

To compare Solar-J and RRTMG-SW, we adopt a standard atmospheric column model, typical of the tropical oceans (surface albedo is 0.06) and define three cases: clear sky, a stratus liquid-water cloud, and a cirrus ice-water cloud. Both cloudy cases assume $100 \%$ cloud cover; the cloud overlap algorithms of Cloud-J are not invoked. Aerosols are not included either. Atmosphere and cloud properties are given in Table 3. Each test case is evaluated at four different solar zenith angles (SZAs) at $0,21,62$, and $84^{\circ}$, whose respective cosine values are $1.0,0.93,0.47$, and 0.10 .

The two cloud profiles are extracted from the 3-hourly, July 2005 ECMWF Integrated Forecast System (IFS) data. This data set has a horizontal resolution of $1^{\circ} \times 1^{\circ}$ in longitude and latitude, and 37 vertical layers with about $0.5 \mathrm{~km}$ vertical resolution in the troposphere. Our example of marine stratus clouds has liquid-water content (LWC, $\mathrm{g} \mathrm{m}^{-3}$ ) only below $2 \mathrm{~km}$, while the cirrus example has non-zero icewater content (IWC, $\mathrm{g} \mathrm{m}^{-3}$ ) above $6 \mathrm{~km}$ and no liquid water anywhere. The total cloud water content (CWC, $\mathrm{g} \mathrm{m}^{-3}$ ) and effective radius $\left(r_{\mathrm{e}}\right)$ are also listed in Table 3 . Solar-J has default values for $r_{\mathrm{e}}$ : for cirrus, they are parameterized as $r_{\mathrm{e}}=164 \times \mathrm{IWC}^{0.23} \mu \mathrm{m}$, based on a fit to the data described in Heymsfield et al. (2003). For liquid-water clouds, they are based loosely on observations of clean maritime stratus (Boers et al., 1998; Gerber, 1996; Miles et al., 2000), with $r_{\mathrm{e}}=9.6 \mu \mathrm{m}$ at pressures greater than $810 \mathrm{hPa}$ and increasing linearly to $12.7 \mu \mathrm{m}$ at $610 \mathrm{hPa}$ and above. When implemented in an atmospheric model, $r_{\mathrm{e}}$ will ideally be supplied by the atmospheric model driving Solar-J.

Heating rates and the changes in the radiative energy budget due to clouds are evaluated with the clear-sky component subtracted. In both Solar-J and RRTMG-SW, when $r_{\mathrm{e}}$ and CWC are given, the corresponding wavelength-dependent properties are derived from tables or formulae. In Solar$\mathrm{J}$, the scattering phase function is truncated at eight terms, but in RRTMG-SW's two-stream model only the first term $\left(A_{1} / 3=g\right)$ is retained. For liquid water, RRTMG-SW adopts the parameterization scheme by Hu and Stamnes (1993). For ice clouds, three different parameterizations are available, and all are tested here (Ebert and Curry, 1992, henceforth EC92; Key, 2002, henceforth Key02; Fu, 1996, henceforth Fu96).

These parameterization schemes in RRTMG-SW aim to fit the ice-cloud optical properties - extinction coefficient, SSA, and $g-$ as a polynomial function of $r_{\mathrm{e}}$ and CWC. Note that Fu's parameterization is based on the generalized effective diameter $\left(D_{\text {ge }}\right)$ but can be related to the input $r_{\mathrm{e}}$ through Eq. (3.12) of Fu (1996). Ebert and Curry's parameterization has been applied in the Community Atmosphere Model (CAM version 4.0 and prior versions). According to the documentation in RRTMG-SW, Key's parameterization was taken from the Mie-calculated spherical shapes of ice particles from the Streamer radiative transfer codes (Key, 2002) and thus should be similar to the Solar-J approximation. The two-stream solution to the radiative transfer problem, as implemented in RRTMG-SW, requires that the scattering optical depth $\left(\tau_{\text {scat }}\right)$ be reduced with what is described as the delta-Eddington approximation (Huang, 1968; Joseph et al., 1976). The purpose is to remove the forward-scattering peak typical of large particles and have only isotropic-equivalent scattering. The absorption optical depth is not changed to ensure correct absorption in the limit of optically thin clouds. The basic problem with these approximations is that the cloud optical depth is reduced by as much as a factor of 5, and thus substantially more sunlight is transmitted through the cloud as a direct solar beam rather than as scattered light. In RRTMG-SW (except for the Fu96 ice-cloud approximation), the HG phase function (Henyey and Greenstein, 1941) is further used to approximate the scattering of aerosols and clouds because of its simple power series formulation. The HG phase function does not represent realistic scattering because it does not have the backward-scattering peak of real aerosols and clouds. As might be expected, errors in twostream approximations are ubiquitous and vary widely with solar zenith angle (Boucher et al., 1998). 
Table 1. Some key configuration parameters for Solar-J v7.5 wavelength bins: solar-flux-weighted wavelength ( $\lambda$ eff) within the range between $\lambda_{\text {beg }}$ and $\lambda_{\text {end }}$, solar fluxes in photons $\mathrm{cm}^{-2} \mathrm{~s}^{-1}\left(S_{\text {phot }}\right)$ and in $\mathrm{W} \mathrm{m}^{-2}\left(S_{\text {watt }}\right)$, Rayleigh cross section $\left(X_{\text {Rayl }}\right)$, and yields for photosynthetically active radiation $\left(Y_{\text {par }}\right)$.

\begin{tabular}{|c|c|c|c|c|c|c|c|}
\hline bin & $\lambda_{\text {eff }}(\mu \mathrm{m})$ & $\lambda_{\text {beg }}(\mu \mathrm{m})$ & $\lambda_{\text {end }}(\mu \mathrm{m})$ & $S_{\text {phot }}\left(\mathrm{cm}^{-2} \mathrm{~s}^{-1}\right)$ & $S_{\text {Watt }}\left(\mathrm{W} \mathrm{m}^{-2}\right)$ & $X_{\text {Rayl }}\left(\mathrm{cm}^{2}\right)$ & $Y_{\text {PAR }}(/$ phot $)$ \\
\hline S01 & 0.187 & & & $1.391 \mathrm{E}+12$ & 0.0147 & $5.073 \mathrm{E}-25$ & \\
\hline S02 & 0.191 & & & $1.627 \mathrm{E}+12$ & 0.0168 & $4.479 \mathrm{E}-25$ & \\
\hline S03 & 0.193 & & & $1.664 \mathrm{E}+12$ & 0.0170 & $4.196 \mathrm{E}-25$ & \\
\hline S04 & 0.196 & & & $9.278 \mathrm{E}+11$ & 0.0094 & $3.906 \mathrm{E}-25$ & \\
\hline S05 & 0.202 & & & $7.842 \mathrm{E}+12$ & 0.0766 & $3.355 \mathrm{E}-25$ & \\
\hline S06 & 0.208 & & & $4.680 \mathrm{E}+12$ & 0.0445 & $2.929 \mathrm{E}-25$ & \\
\hline S07 & 0.211 & & & $9.918 \mathrm{E}+12$ & 0.0930 & $2.736 \mathrm{E}-25$ & \\
\hline S08 & 0.214 & & & $1.219 \mathrm{E}+13$ & 0.1128 & $2.581 \mathrm{E}-25$ & \\
\hline S09 & 0.261 & & & $6.364 \mathrm{E}+14$ & 4.818 & $1.049 \mathrm{E}-25$ & \\
\hline S10 & 0.267 & & & $4.049 \mathrm{E}+14$ & 2.962 & $9.492 \mathrm{E}-26$ & \\
\hline S11 & 0.277 & & & $3.150 \mathrm{E}+14$ & 2.218 & $8.103 E-26$ & \\
\hline S12 & 0.295 & 0.2910 & 0.2982 & $5.893 \mathrm{E}+14$ & 3.703 & $6.135 \mathrm{E}-26$ & \\
\hline S13 & 0.303 & 0.2982 & 0.3074 & $7.670 \mathrm{E}+14$ & 4.670 & $5.424 \mathrm{E}-26$ & \\
\hline S14 & 0.310 & 0.3074 & 0.3124 & $5.041 \mathrm{E}+14$ & 3.063 & $4.925 \mathrm{E}-26$ & \\
\hline S15 & 0.316 & 0.3124 & 0.3203 & $8.895 \mathrm{E}+14$ & 5.414 & $4.516 \mathrm{E}-26$ & \\
\hline S16 & 0.333 & 0.3203 & 0.3450 & $3.852 \mathrm{E}+15$ & 22.28 & $3.644 \mathrm{E}-26$ & 0.0514 \\
\hline S17 & 0.383 & 0.3450 & 0.4124 & $1.547 \mathrm{E}+16$ & 77.17 & $2.082 \mathrm{E}-26$ & 0.4855 \\
\hline S18 & 0.599 & 0.4124 & 0.7780 & $1.805 \mathrm{E}+17$ & 608.68 & $4.427 \mathrm{E}-27$ & 0.6760 \\
\hline S19 & 0.973 & 0.778 & 1.242 & & 349.96 & $5.380 \mathrm{E}-28$ & \\
\hline $\mathrm{S} 20$ & 1.267 & 1.242 & 1.299 & & 25.59 & $1.559 \mathrm{E}-28$ & \\
\hline $\mathrm{S} 21$ & 1.448 & 1.299 & 1.626 & & 102.96 & $9.578 \mathrm{E}-29$ & \\
\hline $\mathrm{S} 22$ & 1.767 & 1.626 & 1.942 & & 56.01 & $4.241 \mathrm{E}-29$ & \\
\hline $\mathrm{S} 23$ & 2.039 & 1.942 & 2.151 & & 22.40 & $2.347 \mathrm{E}-29$ & \\
\hline $\mathrm{S} 24$ & 2.309 & 2.151 & 2.500 & & 23.50 & $1.441 \mathrm{E}-29$ & \\
\hline $\mathrm{S} 25$ & 2.748 & 2.500 & 3.077 & & 20.20 & $7.290 \mathrm{E}-30$ & \\
\hline S26 & 3.404 & 3.077 & 3.846 & & 12.25 & $3.117 \mathrm{E}-30$ & \\
\hline $\mathrm{S} 27$ & 5.362 & 3.846 & 12 & & 12.58 & $8.053 \mathrm{E}-31$ & \\
\hline
\end{tabular}

\section{Results: Solar-J versus RRTMG-SW}

\subsection{Clear sky}

The clear-sky comparison between Solar-J and RRTMG-SW for overhead sun $\left(\mathrm{SZA}=0^{\circ}\right)$ is summarized in Table 4 and Fig. 2. Table 4 lists the band-by-band radiation budget in $\mathrm{W} \mathrm{m}^{-2}$, with Solar-J's spectral bins labeled as S-bins and RRTMG's as B bands (B16-B29 follow the same band numbers as in RRTMG's codes). For easy comparison, several Solar-J's spectral bins of higher resolution from the UV range are lumped together to best match the RRTMG's bin of similar range, and vice versa with RRTMG's B24 and B25 bins combined to compare to Solar-J's S18 bin. The incoming spectral solar irradiance is slightly different for the two codes and so for easier comparison we scale each of them to a total of $1360.8 \mathrm{~W} \mathrm{~m}^{-2}$ (Kopp and Lean, 2011). RRTMG-SW adopts the solar source function from Kurucz (1992), while Solar-J integrates high-resolution $(0.05 \mathrm{~nm})$ photon fluxes (Meier et al., 1992) by wavelength to obtain the solar irradiance. Clear-sky summary comparisons for the other three
SZAs $\left(21,62,84^{\circ}\right)$ are shown in Table 5 under clear-sky columns.

In Table 4, the incoming spectral solar irradiance at top of the atmosphere (TOA down) is balanced by components of (1) the reflected flux going back to space (TOA up positive), (2) the absorption in the atmosphere, separated into the stratosphere and troposphere, and (3) surface heating. Several differences in the configuration of spectral bands between Solar-J and RRTMG-SW affect these results. For one, RRTMG-SW does not include the small amount of solar irradiance at wavelengths $(\lambda)<200 \mathrm{~nm}\left(0.06 \mathrm{~W} \mathrm{~m}^{-2}\right)$ and thus ignores photodissociation of $\mathrm{O}_{2}$ molecules in the Schumann-Runge bands and part of the Herzberg continuum that heats the upper stratosphere and mesosphere. Second, for $\lambda=200-345 \mathrm{~nm}$, Solar-J has $3 \mathrm{~W} \mathrm{~m}^{-2}(6 \%)$ less solar energy than RRTMG-SW and the difference appears in RRTMG's larger heating of the stratosphere. Third, the bin division between 345 and $778 \mathrm{~nm}$ is at $412 \mathrm{~nm}$ for Solar-J (i.e., between S17 and S18), but at $442 \mathrm{~nm}$ for RRTMG-SW (between B26 and B25+B24). This interval, 412-442 nm, has very low $\mathrm{O}_{3}$ absorption, significant Rayleigh scattering, and a large amount of solar energy $\left(\sim 51 \mathrm{~W} \mathrm{~m}^{-2}\right)$. Both the 
Table 2. Spectral properties of liquid-water and ice-water clouds and stratospheric sulfate aerosols: the real and imaginary refractive indices $\left(n_{\mathrm{r}}\right.$ and $\left.n_{\mathrm{i}}\right)$, ratio of optical to geometric cross section $(Q)$, single scattering albedo (SSA), and the asymmetry factor $(g)$.

\begin{tabular}{|c|c|c|c|c|c|c|c|c|c|c|}
\hline \multirow[t]{2}{*}{ Bin } & \multicolumn{5}{|c|}{ Liquid-water cloud: $r_{\mathrm{e}}=12 \mu \mathrm{m}, \rho=1.00 \mathrm{~g} \mathrm{~cm}^{-3}$} & \multicolumn{5}{|c|}{ Ice-water cloud: $r_{\mathrm{e}}=48 \mu \mathrm{m}, \rho=0.917 \mathrm{~g} \mathrm{~cm}^{-3}$} \\
\hline & $n_{\mathrm{r}}$ & $n_{\mathrm{i}}$ & $Q$ & SSA & $g$ & $n_{\mathrm{r}}$ & $n_{\mathrm{i}}$ & $Q$ & SSA & $g$ \\
\hline S12 & 1.350 & $1.8 \mathrm{E}-08$ & 2.054 & 1.0000 & 0.867 & 1.336 & $5.8 \mathrm{E}-09$ & 2.021 & 1.0000 & 0.812 \\
\hline S13 & 1.349 & $1.5 \mathrm{E}-08$ & 2.053 & 1.0000 & 0.869 & 1.333 & $5.4 \mathrm{E}-09$ & 2.021 & 1.0000 & 0.812 \\
\hline S14 & 1.348 & $1.4 \mathrm{E}-08$ & 2.052 & 1.0000 & 0.869 & 1.332 & $5.1 \mathrm{E}-09$ & 2.022 & 1.0000 & 0.812 \\
\hline S15 & 1.347 & $1.3 \mathrm{E}-08$ & 2.055 & 1.0000 & 0.869 & 1.331 & $4.8 \mathrm{E}-09$ & 2.022 & 1.0000 & 0.812 \\
\hline S16 & 1.345 & $9.5 \mathrm{E}-09$ & 2.057 & 1.0000 & 0.869 & 1.328 & $4.3 \mathrm{E}-09$ & 2.023 & 1.0000 & 0.812 \\
\hline S17 & 1.340 & $3.4 \mathrm{E}-09$ & 2.062 & 1.0000 & 0.869 & 1.321 & $3.0 \mathrm{E}-09$ & 2.025 & 1.0000 & 0.812 \\
\hline S18 & 1.333 & $3.1 \mathrm{E}-08$ & 2.089 & 1.0000 & 0.863 & 1.310 & $1.7 \mathrm{E}-08$ & 2.034 & 1.0000 & 0.812 \\
\hline S19 & 1.328 & $2.8 \mathrm{E}-06$ & 2.118 & 0.9996 & 0.858 & 1.302 & $1.7 \mathrm{E}-06$ & 2.047 & 0.9991 & 0.812 \\
\hline S20 & 1.324 & $1.2 \mathrm{E}-05$ & 2.144 & 0.9986 & 0.852 & 1.297 & $1.3 \mathrm{E}-05$ & 2.055 & 0.9946 & 0.812 \\
\hline $\mathrm{S} 21$ & 1.321 & $1.6 \mathrm{E}-04$ & 2.155 & 0.9851 & 0.854 & 1.293 & $2.4 \mathrm{E}-04$ & 2.060 & 0.9246 & 0.812 \\
\hline S22 & 1.313 & $3.2 \mathrm{E}-04$ & 2.179 & 0.9752 & 0.852 & 1.284 & $2.2 \mathrm{E}-04$ & 2.069 & 0.9413 & 0.812 \\
\hline $\mathrm{S} 23$ & 1.302 & $9.2 \mathrm{E}-04$ & 2.197 & 0.9427 & 0.858 & 1.272 & $1.2 \mathrm{E}-03$ & 2.076 & 0.7876 & 0.812 \\
\hline $\mathrm{S} 24$ & 1.283 & $6.7 \mathrm{E}-04$ & 2.220 & 0.9610 & 0.855 & 1.251 & $4.7 \mathrm{E}-04$ & 2.083 & 0.9088 & 0.812 \\
\hline $\mathrm{S} 25$ & 1.239 & $1.0 \mathrm{E}-01$ & 2.211 & 0.4979 & 0.970 & 1.125 & $1.0 \mathrm{E}-01$ & 2.071 & 0.5107 & 0.812 \\
\hline S26 & 1.428 & $5.1 \mathrm{E}-02$ & 2.268 & 0.5240 & 0.939 & 1.496 & $1.6 \mathrm{E}-01$ & 2.102 & 0.5408 & 0.812 \\
\hline S27 & 1.317 & $2.2 \mathrm{E}-02$ & 2.409 & 0.6809 & 0.861 & 1.326 & $2.9 \mathrm{E}-02$ & 2.144 & 0.5245 & 0.812 \\
\hline \multirow[t]{2}{*}{ Bin } & \multicolumn{5}{|c|}{ Strat. sulf., volc.: $r_{\mathrm{e}}=0.422 \mu \mathrm{m}, \rho=1.69 \mathrm{~g} \mathrm{~cm}^{-3}$} & \multicolumn{5}{|c|}{ Strat. sulf., bkgrd: $r_{\mathrm{e}}=0.130 \mu \mathrm{m}, \rho=1.69 \mathrm{~g} \mathrm{~cm}^{-3}$} \\
\hline & $n_{\mathrm{r}}$ & $n_{\mathrm{i}}$ & $Q$ & SSA & $g$ & $n_{\mathrm{r}}$ & $n_{\mathrm{i}}$ & $Q$ & SSA & $g$ \\
\hline S05 & 1.505 & $0.0 \mathrm{E}+00$ & 2.612 & 1.0000 & 0.732 & 1.505 & $0.0 \mathrm{E}+00$ & 2.966 & 1.0000 & 0.698 \\
\hline S06 & 1.505 & $0.0 \mathrm{E}+00$ & 2.638 & 1.0000 & 0.728 & 1.505 & $0.0 \mathrm{E}+00$ & 2.936 & 1.0000 & 0.698 \\
\hline S07 & 1.505 & $0.0 \mathrm{E}+00$ & 2.620 & 1.0000 & 0.735 & 1.505 & $0.0 \mathrm{E}+00$ & 2.919 & 1.0000 & 0.699 \\
\hline S08 & 1.505 & $0.0 \mathrm{E}+00$ & 2.628 & 1.0000 & 0.734 & 1.505 & $0.0 \mathrm{E}+00$ & 2.904 & 1.0000 & 0.699 \\
\hline S09 & 1.472 & $0.0 \mathrm{E}+00$ & 2.604 & 1.0000 & 0.718 & 1.472 & $0.0 \mathrm{E}+00$ & 2.435 & 1.0000 & 0.711 \\
\hline S10 & 1.469 & $0.0 \mathrm{E}+00$ & 2.606 & 1.0000 & 0.710 & 1.469 & $0.0 \mathrm{E}+00$ & 2.379 & 1.0000 & 0.711 \\
\hline S11 & 1.464 & $0.0 \mathrm{E}+00$ & 2.556 & 1.0000 & 0.707 & 1.464 & $0.0 \mathrm{E}+00$ & 2.271 & 1.0000 & 0.711 \\
\hline $\mathrm{S} 12$ & 1.456 & $0.0 \mathrm{E}+00$ & 2.500 & 1.0000 & 0.695 & 1.456 & $0.0 \mathrm{E}+00$ & 2.087 & 1.0000 & 0.709 \\
\hline S13 & 1.452 & $0.0 \mathrm{E}+00$ & 2.474 & 1.0000 & 0.690 & 1.452 & $0.0 \mathrm{E}+00$ & 1.998 & 1.0000 & 0.708 \\
\hline S14 & 1.451 & $0.0 \mathrm{E}+00$ & 2.461 & 1.0000 & 0.686 & 1.451 & $0.0 \mathrm{E}+00$ & 1.940 & 1.0000 & 0.706 \\
\hline S15 & 1.451 & $0.0 \mathrm{E}+00$ & 2.449 & 1.0000 & 0.683 & 1.451 & $0.0 \mathrm{E}+00$ & 1.892 & 1.0000 & 0.704 \\
\hline S16 & 1.450 & $0.0 \mathrm{E}+00$ & 2.432 & 1.0000 & 0.676 & 1.450 & $0.0 \mathrm{E}+00$ & 1.766 & 1.0000 & 0.698 \\
\hline S17 & 1.445 & $0.0 \mathrm{E}+00$ & 2.475 & 1.0000 & 0.675 & 1.445 & $0.0 \mathrm{E}+00$ & 1.432 & 1.0000 & 0.683 \\
\hline S18 & 1.431 & $1.7 \mathrm{E}-08$ & 3.017 & 1.0000 & 0.723 & 1.431 & $1.7 \mathrm{E}-08$ & 0.620 & 1.0000 & 0.593 \\
\hline S19 & 1.424 & $1.5 \mathrm{E}-06$ & 2.212 & 1.0000 & 0.663 & 1.424 & $1.5 \mathrm{E}-06$ & 0.193 & 1.0000 & 0.434 \\
\hline S20 & 1.417 & $8.6 \mathrm{E}-06$ & 1.431 & 0.9999 & 0.605 & 1.417 & $8.6 \mathrm{E}-06$ & 0.090 & 0.9998 & 0.336 \\
\hline $\mathrm{S} 21$ & 1.430 & $9.4 \mathrm{E}-05$ & 1.173 & 0.9988 & 0.570 & 1.430 & $9.4 \mathrm{E}-05$ & 0.065 & 0.9972 & 0.291 \\
\hline S22 & 1.422 & $4.7 \mathrm{E}-04$ & 0.724 & 0.9910 & 0.511 & 1.422 & $4.7 \mathrm{E}-04$ & 0.033 & 0.9782 & 0.225 \\
\hline $\mathrm{S} 23$ & 1.410 & $1.3 \mathrm{E}-03$ & 0.475 & 0.9672 & 0.456 & 1.410 & $1.3 \mathrm{E}-03$ & 0.021 & 0.9184 & 0.182 \\
\hline $\mathrm{S} 24$ & 1.388 & $2.1 \mathrm{E}-03$ & 0.305 & 0.9288 & 0.397 & 1.388 & $2.1 \mathrm{E}-03$ & 0.013 & 0.8166 & 0.148 \\
\hline $\mathrm{S} 25$ & 1.319 & $5.1 \mathrm{E}-02$ & 0.253 & 0.3855 & 0.302 & 1.319 & $5.1 \mathrm{E}-02$ & 0.040 & 0.0768 & 0.106 \\
\hline S26 & 1.366 & $1.7 \mathrm{E}-01$ & 0.424 & 0.1714 & 0.214 & 1.366 & $1.7 \mathrm{E}-01$ & 0.098 & 0.0219 & 0.074 \\
\hline S27 & 1.406 & $2.1 \mathrm{E}-01$ & 0.274 & 0.0744 & 0.091 & 1.406 & $2.1 \mathrm{E}-01$ & 0.073 & 0.0066 & 0.033 \\
\hline
\end{tabular}

shorter-wavelength bins (S17 or B26) reflect about $20 \%$ of the incoming radiation, but in the adjacent bin with the Chappuis $\mathrm{O}_{3}$ band it is only about $9 \%$. Thus, placing the $412-$ $442 \mathrm{~nm}$ interval with the Chappuis band results in greater atmospheric absorption and less reflection. The benefit of moving the Solar-J (and Cloud-J) band edge to $442 \mathrm{~nm}$ should be investigated.
These differences, particularly the $412-442 \mathrm{~nm}$ interval, explain most of the total budget difference where, overall, Solar-J reflects $4 \mathrm{~W} \mathrm{~m}^{-2}$ (4\%) less back to space, absorbs $2 \mathrm{~W} \mathrm{~m}^{-2}(6 \%)$ less in the stratosphere, $3 \mathrm{~W} \mathrm{~m}^{-2}(1 \%)$ more in the troposphere, and $3 \mathrm{~W} \mathrm{~m}^{-2}(1 / 2 \%)$ more at the surface. For SZAs of 21 and $62^{\circ}$ (Table 5), Solar-J continues to reflect 3-4 $\mathrm{W} \mathrm{m}^{-2}$ less energy back to space, but at a large SZA of 
Table 3. The standard tropical atmosphere and the two cloud profiles implemented in both Solar-J and RRTMG-SW. Height (Z) and pressure $(P)$ are edge values; others are layer averages.

\begin{tabular}{|c|c|c|c|c|c|c|c|c|c|}
\hline Layer & $\begin{array}{r}Z_{\text {edge }} \\
(\mathrm{km})\end{array}$ & $\begin{array}{l}P_{\text {edge }} \\
(\mathrm{hPa})\end{array}$ & $\begin{array}{r}T \\
(\mathrm{~K})\end{array}$ & $\begin{array}{r}\mathrm{O}_{3} \\
\left(\mathrm{~cm}^{-3}\right)\end{array}$ & $\begin{array}{r}\mathrm{H}_{2} \mathrm{O} \\
\left(\mathrm{kg} \mathrm{kg}^{-1}\right)\end{array}$ & $\begin{array}{l}\text { Stratus cloud } \\
\mathrm{LWC}\left(\mathrm{g} \mathrm{m}^{-3}\right)\end{array}$ & $\begin{array}{l}\text { LWC } \\
r_{\mathrm{e}}(\mu \mathrm{m})\end{array}$ & $\begin{array}{l}\text { Cirrus clou } \\
\text { IWC }\left(\mathrm{g} \mathrm{m}^{-3}\right.\end{array}$ & $\begin{array}{l}\text { IWC } \\
\mathrm{e}(\mu \mathrm{m})\end{array}$ \\
\hline 58 & 75.25 & 0.020 & & & & & & & \\
\hline 57 & 59.58 & 0.200 & 232.4 & $1.27 \mathrm{E}+09$ & $3.85 \mathrm{e}-06$ & 0 & 0 & 0 & 0 \\
\hline 56 & 54.95 & 0.384 & 242.4 & $1.17 \mathrm{E}+10$ & $3.85 \mathrm{e}-06$ & 0 & 0 & 0 & 0 \\
\hline 55 & 51.11 & 0.636 & 259.9 & $2.81 \mathrm{E}+10$ & $3.85 \mathrm{e}-06$ & 0 & 0 & 0 & 0 \\
\hline 54 & 47.91 & 0.956 & 268.1 & $5.79 \mathrm{E}+10$ & $3.82 \mathrm{e}-06$ & 0 & 0 & 0 & 0 \\
\hline 53 & 45.25 & 1.345 & 266.9 & $1.07 \mathrm{E}+11$ & $3.78 \mathrm{e}-06$ & 0 & 0 & 0 & 0 \\
\hline 52 & 42.97 & 1.806 & 263.9 & $1.84 \mathrm{E}+11$ & $3.63 e-06$ & 0 & 0 & 0 & 0 \\
\hline 51 & 40.97 & 2.348 & 259.9 & $3.01 \mathrm{E}+11$ & $3.42 \mathrm{e}-06$ & 0 & 0 & 0 & 0 \\
\hline 50 & 39.18 & 2.985 & 255.2 & $4.66 \mathrm{E}+11$ & $3.20 \mathrm{e}-06$ & 0 & 0 & 0 & 0 \\
\hline 49 & 37.52 & 3.740 & 250.7 & $6.78 \mathrm{E}+11$ & $3.00 \mathrm{e}-06$ & 0 & 0 & 0 & 0 \\
\hline 48 & 35.96 & 4.646 & 245.1 & $9.63 \mathrm{E}+11$ & $2.74 \mathrm{e}-06$ & 0 & 0 & 0 & 0 \\
\hline 47 & 34.46 & 5.757 & 240.3 & $1.30 \mathrm{E}+12$ & $2.55 \mathrm{e}-06$ & 0 & 0 & 0 & 0 \\
\hline 46 & 32.97 & 7.132 & 237.2 & $1.70 \mathrm{E}+12$ & $2.47 \mathrm{e}-06$ & 0 & 0 & 0 & 0 \\
\hline 45 & 31.50 & 8.837 & 234.3 & $2.20 \mathrm{E}+12$ & $2.42 \mathrm{e}-06$ & 0 & 0 & 0 & 0 \\
\hline 44 & 30.04 & 10.95 & 231.6 & $2.87 \mathrm{E}+12$ & $2.27 \mathrm{e}-06$ & 0 & 0 & 0 & 0 \\
\hline 43 & 28.61 & 13.57 & 228.7 & $3.56 \mathrm{E}+12$ & $2.18 \mathrm{e}-06$ & 0 & 0 & 0 & 0 \\
\hline 42 & 27.20 & 16.81 & 225.2 & $4.24 \mathrm{E}+12$ & $2.11 \mathrm{e}-06$ & 0 & 0 & 0 & 0 \\
\hline 41 & 25.81 & 20.82 & 221.4 & $4.88 \mathrm{E}+12$ & $2.04 \mathrm{e}-06$ & 0 & 0 & 0 & 0 \\
\hline 40 & 24.45 & 25.80 & 216.0 & $4.67 \mathrm{E}+12$ & $1.90 \mathrm{e}-06$ & 0 & 0 & 0 & 0 \\
\hline 39 & 23.12 & 31.96 & 211.9 & $4.36 \mathrm{E}+12$ & $1.87 \mathrm{e}-06$ & 0 & 0 & 0 & 0 \\
\hline 38 & 21.80 & 39.60 & 211.4 & $3.93 \mathrm{E}+12$ & $1.85 \mathrm{e}-06$ & 0 & 0 & 0 & 0 \\
\hline 37 & 20.48 & 49.07 & 209.8 & $3.31 \mathrm{E}+12$ & $1.84 \mathrm{e}-06$ & 0 & 0 & 0 & 0 \\
\hline 36 & 19.25 & 60.18 & 205.9 & $2.01 \mathrm{E}+12$ & $1.90 \mathrm{e}-06$ & 0 & 0 & 0 & 0 \\
\hline 35 & 18.10 & 73.07 & 202.2 & $1.47 \mathrm{E}+12$ & $2.04 \mathrm{e}-06$ & 0 & 0 & 0 & 0 \\
\hline 34 & 17.05 & 87.73 & 196.6 & $1.02 \mathrm{E}+12$ & $3.27 \mathrm{e}-06$ & 0 & 0 & $1.10 \mathrm{E}-06$ & 6.99 \\
\hline 33 & 16.08 & 104.2 & 191.1 & $4.10 \mathrm{E}+11$ & $3.49 \mathrm{e}-06$ & 0 & 0 & $5.88 \mathrm{E}-05$ & 17.45 \\
\hline 32 & 15.17 & 122.6 & 192.1 & $4.06 \mathrm{E}+11$ & $4.76 \mathrm{e}-06$ & 0 & 0 & $1.32 \mathrm{E}-04$ & 21.03 \\
\hline 31 & 14.28 & 142.8 & 197.6 & $3.25 \mathrm{E}+11$ & $9.07 \mathrm{e}-06$ & 0 & 0 & $3.49 \mathrm{E}-04$ & 26.29 \\
\hline 30 & 13.43 & 165.0 & 203.6 & $3.28 \mathrm{E}+11$ & $1.83 \mathrm{e}-05$ & 0 & 0 & $8.40 \mathrm{E}-04$ & 32.17 \\
\hline 29 & 12.59 & 188.9 & 209.8 & $3.23 \mathrm{E}+11$ & $3.54 \mathrm{e}-05$ & 0 & 0 & $1.02 \mathrm{E}-03$ & 33.66 \\
\hline 28 & 11.78 & 214.6 & 216.6 & $3.45 \mathrm{E}+11$ & $6.38 \mathrm{e}-05$ & 0 & 0 & $1.46 \mathrm{E}-03$ & 36.54 \\
\hline 27 & 11.00 & 242.1 & 223.4 & $3.55 \mathrm{E}+11$ & $1.12 \mathrm{e}-04$ & 0 & 0 & $2.01 \mathrm{E}-03$ & 39.31 \\
\hline 26 & 10.23 & 271.2 & 230.0 & $3.88 \mathrm{E}+11$ & $1.95 \mathrm{e}-04$ & 0 & 0 & $2.19 \mathrm{E}-03$ & 40.12 \\
\hline 25 & 9.48 & 302.1 & 236.2 & $4.29 \mathrm{E}+11$ & $3.21 \mathrm{e}-04$ & 0 & 0 & $3.41 \mathrm{E}-03$ & 44.39 \\
\hline 24 & 8.76 & 334.6 & 242.4 & $4.66 \mathrm{E}+11$ & $4.94 \mathrm{e}-04$ & 0 & 0 & $1.92 \mathrm{E}-04$ & 22.90 \\
\hline 23 & 8.06 & 368.6 & 248.2 & $5.02 \mathrm{E}+11$ & $7.14 \mathrm{e}-04$ & 0 & 0 & $3.35 \mathrm{E}-04$ & 26.03 \\
\hline 22 & 7.38 & 403.9 & 253.4 & $5.40 \mathrm{E}+11$ & $9.80 \mathrm{e}-04$ & 0 & 0 & $1.85 \mathrm{E}-05$ & 13.38 \\
\hline 21 & 6.73 & 440.3 & 258.1 & $5.80 \mathrm{E}+11$ & $1.31 \mathrm{e}-03$ & 0 & 0 & $2.59 \mathrm{E}-07$ & 5.01 \\
\hline 20 & 6.10 & 477.5 & 262.2 & $6.21 \mathrm{E}+11$ & $1.78 \mathrm{e}-03$ & 0 & 0 & $8.58 \mathrm{E}-08$ & 3.89 \\
\hline 19 & 5.51 & 515.4 & 266.0 & $6.22 \mathrm{E}+11$ & $2.37 \mathrm{e}-03$ & 0 & 0 & $2.13 \mathrm{E}-07$ & 4.79 \\
\hline 18 & 4.94 & 553.7 & 269.6 & $6.46 \mathrm{E}+11$ & $3.13 e-03$ & 0 & 0 & $5.98 \mathrm{E}-08$ & 3.58 \\
\hline 17 & 4.41 & 591.9 & 272.7 & $6.84 \mathrm{E}+11$ & $4.02 \mathrm{e}-03$ & 0 & 0 & 0 & 0 \\
\hline 16 & 3.91 & 629.9 & 275.3 & $7.23 \mathrm{E}+11$ & $5.14 \mathrm{e}-03$ & 0 & 0 & 0 & 0 \\
\hline 15 & 3.44 & 667.2 & 278.0 & $6.80 \mathrm{E}+11$ & $6.31 \mathrm{e}-03$ & 0 & 0 & 0 & 0 \\
\hline 14 & 3.00 & 703.7 & 280.8 & $6.19 \mathrm{E}+11$ & $7.49 \mathrm{e}-03$ & 0 & 0 & 0 & 0 \\
\hline 13 & 2.60 & 738.9 & 282.9 & $6.46 \mathrm{E}+11$ & $8.67 \mathrm{e}-03$ & 0 & 0 & 0 & 0 \\
\hline 12 & 2.22 & 772.7 & 284.9 & $6.72 \mathrm{E}+11$ & $1.00 \mathrm{e}-02$ & 0 & 0 & 0 & 0 \\
\hline 11 & 1.88 & 804.6 & 286.9 & $6.97 \mathrm{E}+11$ & $1.17 \mathrm{e}-02$ & $2.66 \mathrm{E}-02$ & 9.60 & 0 & 0 \\
\hline 10 & 1.57 & 834.6 & 288.6 & $7.20 \mathrm{E}+11$ & $1.35 \mathrm{e}-02$ & $2.05 \mathrm{E}-02$ & 9.60 & 0 & 0 \\
\hline 9 & 1.30 & 862.3 & 290.3 & $7.41 \mathrm{E}+11$ & $1.45 \mathrm{e}-02$ & $8.66 \mathrm{E}-02$ & 9.60 & 0 & 0 \\
\hline 8 & 1.05 & 887.6 & 291.8 & $6.30 \mathrm{E}+11$ & $1.54 \mathrm{e}-02$ & $1.21 \mathrm{E}-01$ & 9.60 & 0 & 0 \\
\hline 7 & 0.83 & 910.3 & 293.0 & $6.22 \mathrm{E}+11$ & $1.62 \mathrm{e}-02$ & $9.67 \mathrm{E}-02$ & 9.60 & 0 & 0 \\
\hline 6 & 0.64 & 930.3 & 294.2 & $6.34 \mathrm{E}+11$ & $1.71 \mathrm{e}-02$ & $4.22 \mathrm{E}-02$ & 9.60 & 0 & 0 \\
\hline 5 & 0.49 & 947.7 & 295.3 & $6.45 \mathrm{E}+11$ & $1.79 \mathrm{e}-02$ & $1.53 \mathrm{E}-02$ & 9.60 & 0 & 0 \\
\hline 4 & 0.35 & 962.3 & 296.1 & $6.54 \mathrm{E}+11$ & $1.84 \mathrm{e}-02$ & $6.62 \mathrm{E}-03$ & 9.60 & 0 & 0 \\
\hline 3 & 0.25 & 974.3 & 296.7 & $6.62 \mathrm{E}+11$ & $1.89 \mathrm{e}-02$ & $3.01 \mathrm{E}-03$ & 9.60 & 0 & 0 \\
\hline 2 & 0.10 & 990.9 & 297.7 & $6.69 \mathrm{E}+11$ & $1.98 \mathrm{e}-02$ & $5.69 \mathrm{E}-04$ & 9.60 & 0 & 0 \\
\hline 1 & 0.00 & 1002.0 & 298.9 & $6.76 \mathrm{E}+11$ & $2.09 \mathrm{e}-02$ & $1.56 \mathrm{E}-04$ & 9.60 & 0 & 0 \\
\hline
\end{tabular}


Table 4. Spectral shortwave radiation energy budget in $\mathrm{W} \mathrm{m}^{-2}$ under clear aerosol-free July tropical conditions: Solar-J versus RRTMG. The solar constant is set at $1360.8 \mathrm{~W} \mathrm{~m}^{-2}$. For easy comparison, some Solar-J bins are combined to best match RRTMG's band of similar range and vice versa. Note that the atmospheric absorption is further split into the stratospheric and tropospheric components (see numbers in italics).

\begin{tabular}{lrrrrr}
\hline \multicolumn{5}{c}{ (a) Clear-sky solar radiation budget comparison $\left(\mathrm{W} \mathrm{m}^{-2}\right)$} \\
\hline Solar-J S-bins & $\mathrm{S} 1-\mathrm{S} 4$ & $\mathrm{~S} 5-\mathrm{S} 9$ & $\mathrm{~S} 10-\mathrm{S} 16$ & $\mathrm{~S} 17$ & $\mathrm{~S} 18$ \\
\hline$\lambda(\mathrm{nm})$ & $177-200$ & $200-275$ & $275-345$ & $345-412$ & $412-778$ \\
TOA (down) & 0.06 & 5.14 & 44.31 & 77.17 & 608.68 \\
TOA (up) & 0.00 & 0.01 & 7.52 & 16.89 & 54.36 \\
Atmosphere & 0.06 & 5.14 & 18.01 & 0.05 & 31.96 \\
$\quad$-Stratosphere & 0.06 & 5.14 & 16.97 & 0.04 & 9.41 \\
-Troposphere & 0.00 & 0.00 & 1.04 & 0.01 & 22.55 \\
Surface & 0.00 & 0.00 & 18.78 & 60.23 & 522.43 \\
\hline RRTMG bands & $/$ & $\mathrm{B} 28$ & $\mathrm{~B} 27$ & $\mathrm{~B} 26$ & $\mathrm{~B} 25+\mathrm{B} 24$ \\
$\lambda$ (nm) & $/$ & $200-263$ & $263-345$ & $345-442$ & $442-778$ \\
TOA (down) & $/$ & 3.06 & 49.88 & 128.79 & 562.34 \\
TOA (up) & $/$ & 0.02 & 7.37 & 25.75 & 50.44 \\
Atmosphere & $/$ & 3.05 & 23.24 & 0.00 & 28.77 \\
$\quad$-Stratosphere & $/$ & 3.04 & 22.11 & 0.00 & 8.60 \\
-Troposphere & $/$ & 0.01 & 1.13 & 0.00 & 20.17 \\
Surface & $/$ & 0.00 & 19.29 & 103.04 & 483.13 \\
\end{tabular}

(b) Clear-sky solar radiation budget comparison $\left(\mathrm{W} \mathrm{m}^{-2}\right)$

\begin{tabular}{lrrrrr}
\hline Solar-J S-bins & S19 & S20 & S21 & S22 & S23 \\
\hline$\lambda(\mu \mathrm{m})$ & $0.78-1.24$ & $1.24-1.30$ & $1.30-1.63$ & $1.63-1.94$ & $1.94-2.15$ \\
TOA (down) & 349.96 & 25.59 & 102.96 & 56.01 & 22.40 \\
TOA (up) & 15.33 & 1.28 & 2.17 & 1.43 & 0.6 \\
Atmosphere & 87.94 & 2.36 & 60.53 & 29.41 & 9.48 \\
$\quad$-Stratosphere & 0.00 & 0.12 & 0.29 & 0.20 & 0.41 \\
$\quad$-Troposphere & 87.95 & 2.23 & 60.24 & 29.21 & 9.07 \\
Surface & 246.69 & 21.96 & 40.25 & 25.17 & 12.32 \\
\hline RRTMG bands & $\mathrm{B} 23$ & $\mathrm{~B} 22$ & $\mathrm{~B} 21$ & $\mathrm{~B} 20$ & $\mathrm{~B} 19$ \\
$\lambda$ ( $\mu$ m) & $0.78-1.24$ & $1.24-1.30$ & $1.30-1.63$ & $1.63-1.94$ & $1.94-2.15$ \\
TOA (down) & 343.86 & 24.16 & 102.37 & 55.32 & 22.31 \\
TOA (up) & 14.91 & 1.20 & 2.03 & 1.40 & 0.57 \\
Atmosphere & 86.60 & 2.22 & 61.73 & 29.09 & 9.52 \\
-Stratosphere & 0.00 & 0.12 & 0.30 & 0.20 & 0.42 \\
$\quad$-Troposphere & 86.60 & 2.10 & 61.43 & 28.89 & 9.11 \\
Surface & 242.35 & 20.74 & 38.61 & 24.85 & 12.22
\end{tabular}

(c) Clear-sky solar radiation budget comparison $\left(\mathrm{W} \mathrm{m}^{-2}\right)$

\begin{tabular}{lrrrrr}
\hline Solar-J S-bins & S24 & S25 & S26 & S27 & All bands \\
$\lambda(\mathrm{nm})$ & $2.15-2.50$ & $2.50-3.08$ & $3.08-3.85$ & $3.85-12$ & $0.18-12$ \\
TOA (down) & 23.50 & 20.20 & 12.25 & 12.58 & 1360.80 \\
TOA (up) & 0.75 & 0.00 & 0.19 & 0.05 & 100.53 \\
Atmosphere & 8.32 & 20.17 & 7.28 & 10.36 & 291.06 \\
$\quad$-Stratosphere & 0.07 & 1.65 & 0.17 & 1.28 & 35.80 \\
$\quad$-Troposphere & 8.25 & 18.52 & 7.11 & 9.08 & 255.26 \\
Surface & 14.43 & 0.03 & 4.78 & 2.17 & 969.23 \\
\hline RRTMG bands & $\mathrm{B} 18$ & $\mathrm{~B} 17$ & $\mathrm{~B} 16$ & $\mathrm{~B} 29$ & All bands \\
\hline$\lambda$ (nm) & $2.15-2.50$ & $2.50-3.08$ & $3.08-3.85$ & $3.85-12$ & $0.20-12$ \\
TOA (down) & 23.60 & 20.25 & 12.04 & 12.82 & 1360.80 \\
TOA (up) & 0.76 & 0.00 & 0.18 & 0.05 & 104.45 \\
Atmosphere & 7.96 & 20.22 & 7.17 & 10.57 & 290.27 \\
$\quad$-Stratosphere & 0.06 & 1.66 & 0.16 & 1.30 & 37.91 \\
$\quad$-Troposphere & 7.84 & 18.56 & 7.01 & 9.26 & 252.36 \\
Surface & 14.88 & 0.03 & 4.69 & 2.22 & 966.08 \\
\hline
\end{tabular}



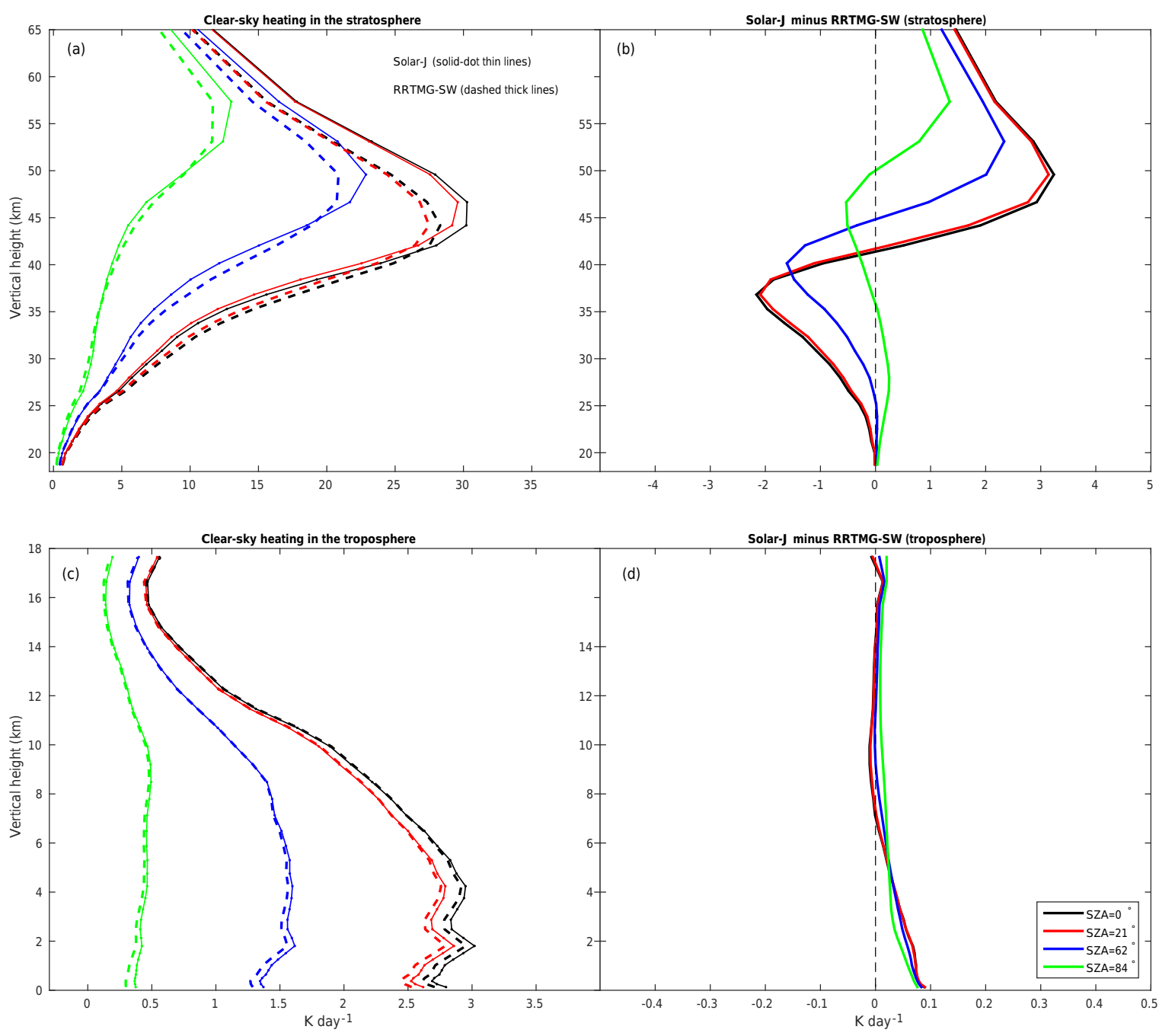

Figure 2. Aerosol-free cloudless atmospheric heating profiles of Solar-J (solid lines) and RRTMG-SW (dashed lines) and the difference, Solar-J minus RRTMG-SW, for a typical July tropical atmosphere at four solar zenith angles with Lambertian surface albedo of 0.06 (left and right sides). The plot is further split into the stratosphere and the troposphere (top and bottom rows). Note that the scale of the $x$ axis, $\mathrm{Kday}^{-1}$, is 10 times larger for the stratosphere on the top panels.

$84^{\circ}$ the two models match closely. While spherical effects may play some role in this shift, we suspect that Rayleigh scattering may contribute. The forward-backward enhancement in Rayleigh scattering is not represented in two-stream isotropic scattering. Thus, RRTMG - Solar-J differences will shift as the primary beam shifts from vertical to horizontal as a much greater fraction of the visible light is scattered. At low sun, the Rayleigh optical slant path along the solar beam is much greater than 1 for bin S17 and even $\sim 1$ for S18.

Figure 2 compares the vertical profiles of clear-sky heating rates $\left(\mathrm{K} \mathrm{day}^{-1}\right)$ for overhead sun $\left(\mathrm{SZA}=0^{\circ}\right)$ with the abscissa axis scaled separately for the stratosphere and the troposphere. Both models produce similar structures with the heating maximum of $\sim 30 \mathrm{Kday}^{-1}$ in the stratosphere at about $45 \mathrm{~km}$ altitude and $\sim 3 \mathrm{Kday}^{-1}$ in the lower troposphere. The ability of Solar-J to match these structures demonstrates that Solar-J has correctly implemented the RRTMG-SW spectral model. Solar-J minus RRTMG-SW differences are shown in the right two panels of Fig. 2. As shown in Fig. 2d, the consistent difference of $<0.1 \mathrm{~K} \mathrm{day}^{-1}$ near the surface come from Solar-J's simplification of combing RRTMG's 14 sub-bins with $\mathrm{O}_{2}$ and $\mathrm{H}_{2} \mathrm{O}$ absorption in bins B24-B25 into the five sub-bins of S18. In the troposphere these are small, but in the stratosphere there is a clear bias with Solar-J producing more heating above 40 $50 \mathrm{~km}$ and less heating below. Differences at the top, above $50 \mathrm{~km}$, are due in part to the lack of $\lambda<200 \mathrm{~nm}$ radiation in RRTMG-SW, and in part due to a better resolution of the $\mathrm{O}_{3}$ and $\mathrm{O}_{2}$ cross sections in Solar-J. Overall, RRTMG-SW deposits more energy in the lower stratosphere, below $35 \mathrm{~km}$, except at larger SZA where it deposits less. Thus, for the tropics and midlatitudes, RRTMG will overheat the lower 
Table 5. Radiation budget comparison $\left(\mathrm{W} \mathrm{m}^{-2}\right.$ ) between Solar-J and RRTMG-SW for top of atmosphere (TOA), atmosphere, and surface across four SZAs. Also shown is the cloud radiative effect (CRE) of a typical marine stratus cloud, for which the atmospheric absorption is split into above cloud, in cloud, and below cloud, and the values are shown in italics.

\begin{tabular}{|c|c|c|c|c|c|c|c|c|}
\hline SZA & \multicolumn{2}{|c|}{$0^{\circ}$} & \multicolumn{2}{|c|}{$21.2^{\circ}$} & \multicolumn{2}{|c|}{$62.2^{\circ}$} & \multicolumn{2}{|c|}{$84.0^{\circ}$} \\
\hline Flux $\left(\mathrm{W} \mathrm{m}^{-2}\right)$ & \multicolumn{2}{|c|}{1360.8} & \multicolumn{2}{|c|}{1268.4} & \multicolumn{2}{|c|}{634.2} & \multicolumn{2}{|c|}{149.1} \\
\hline \multicolumn{9}{|c|}{ Clear-sky radiation budget $\left(\mathrm{W} \mathrm{m}^{-2}\right)$} \\
\hline & Solar-J & RRTMG & Solar-J & RRTMG & Solar-J & RRTMG & Solar-J & RRTMG \\
\hline TOA (up) & 100.5 & 104.5 & 96.2 & 100.3 & 63.9 & 67.3 & 28.2 & 28.5 \\
\hline Atmosphere (absorbed) & 291.1 & 290.2 & 276.6 & 275.3 & 166.5 & 164.0 & 56.6 & 54.9 \\
\hline Surface (absorbed) & 969.2 & 966.1 & 895.7 & 892.8 & 403.8 & 402.9 & 64.3 & 65.6 \\
\hline \multicolumn{9}{|c|}{ Cloud radiative effect (CRE) of a marine stratus cloud $\left(\mathrm{W} \mathrm{m}^{-2}\right)$} \\
\hline & Solar-J & RRTMG & Solar-J & RRTMG & Solar-J & RRTMG & Solar-J & RRTMG \\
\hline TOA & +473.4 & +459.4 & 452.7 & +441.2 & +262.7 & +255.8 & +52.2 & +50.3 \\
\hline Atmosphere & +87.8 & +88.2 & +77.7 & +78.2 & +20.3 & +18.5 & -3.2 & -3.3 \\
\hline Above cloud & +21.1 & +23.8 & +17.7 & +22.8 & +11.3 & +12.0 & +3.0 & +1.6 \\
\hline In cloud & +77.5 & +73.7 & +70.5 & +64.7 & +16.1 & +12.9 & -3.9 & -3.0 \\
\hline Below cloud & -10.8 & -9.3 & -10.6 & -9.2 & -7.1 & -6.4 & -2.2 & -1.8 \\
\hline Surface & -561.2 & -547.6 & -530.4 & -519.4 & -282.9 & -274.4 & -49.1 & -47.0 \\
\hline
\end{tabular}

Table 6. Cirrus ice cloud optical properties: total optical depth $\tau$ for Solar-J and delta-scaled $\tau^{\prime}$ for RRTMG-SW, asymmetry factor $g$, and absorption optical depth, $\tau_{\mathrm{abs}}$, for bins S18 to S27. See Table 1 for wavelength ranges and RRTMG-SW-equivalent bins.

\begin{tabular}{lrrrrrrrrrr}
\hline S-bins & S18 & S19 & S20 & S21 & S22 & S23 & S24 & S25 & S26 & S27 \\
\hline$\lambda_{\text {eff }}$ & $599 \mathrm{~nm}$ & $973 \mathrm{~nm}$ & $1.27 \mu \mathrm{m}$ & $1.45 \mu \mathrm{m}$ & $1.77 \mu \mathrm{m}$ & $2.04 \mu \mathrm{m}$ & $2.31 \mu \mathrm{m}$ & $2.75 \mu \mathrm{m}$ & $3.40 \mu \mathrm{m}$ & $5.36 \mu \mathrm{m}$ \\
\hline \multicolumn{7}{c}{ Total optical depth $\left(\tau\right.$ for Solar-J and reduced $\tau^{\prime}$ for RRTMG schemes $)$} \\
\hline Solar-J & 0.4287 & 0.4322 & 0.4345 & 0.4360 & 0.4383 & 0.4404 & 0.4425 & 0.4380 & 0.4470 & 0.4591 \\
EC92 & 0.2488 & 0.2462 & 0.2462 & 0.2385 & 0.2385 & 0.2276 & 0.2276 & 0.3313 & 0.3313 & 0.3313 \\
Fu96 & 0.1535 & 0.1581 & 0.1563 & 0.1627 & 0.1640 & 0.1575 & 0.1932 & 0.3709 & 0.3382 & 0.3177 \\
Key02 & 0.0923 & 0.0943 & 0.0950 & 0.1041 & 0.1032 & 0.1277 & 0.1065 & 0.1783 & 0.2159 & 0.2266 \\
\hline & & & & Asymmetry factor, $g=A 1 / 3$ & & & \\
\hline Solar-J & 0.7643 & 0.7642 & 0.7641 & 0.7640 & 0.7639 & 0.7639 & 0.7638 & 0.7639 & 0.7635 & 0.7631 \\
EC92 & 0.4406 & 0.4425 & 0.4425 & 0.4484 & 0.4484 & 0.4579 & 0.4579 & 0.4907 & 0.4907 & 0.4907 \\
Fu96 & 0.4591 & 0.4680 & 0.4803 & 0.4987 & 0.5168 & 0.5670 & 0.5870 & 0.6744 & 0.3411 & 0.0000 \\
Key02 & 0.4694 & 0.4692 & 0.4691 & 0.4707 & 0.4707 & 0.4757 & 0.4731 & 0.4866 & 0.4807 & 0.4858 \\
\hline & & & & Total absorbing optical depth $(\tau$ abs $)$ & & & \\
\hline Solar-J & 0.0000 & 0.0003 & 0.0018 & 0.0257 & 0.0201 & 0.0758 & 0.0317 & 0.2163 & 0.2075 & 0.2126 \\
EC92 & 0.0000 & 0.0004 & 0.0004 & 0.0247 & 0.0247 & 0.0558 & 0.0558 & 0.3063 & 0.3063 & 0.3063 \\
Fu96 & 0.0000 & 0.0003 & 0.0022 & 0.0232 & 0.0231 & 0.0743 & 0.0289 & 0.1294 & 0.1743 & 0.1972 \\
Key02 & 0.0000 & 0.0001 & 0.0011 & 0.0178 & 0.0162 & 0.0619 & 0.0290 & 0.1491 & 0.1788 & 0.1983 \\
\hline
\end{tabular}

stratosphere, possibly changing the stability and wave propagation to the high latitudes (Hsu et al., 2013). At high latitudes, RRTMG error is in the opposite direction, resulting in a colder polar stratosphere with possibly stronger winter vortices.

Solar-J traces the solar beam through a spherical atmosphere back to the sun. RRTMG assumes a flat Earth. Both then calculate the subsequent scattering and absorption in a plane-parallel, flat atmosphere, but with different solar source terms at each level. Solar-J is able to simulate both photolysis and heating rates throughout twilight, even when the sun is no longer directly visible at the layer. Figure 3a shows the smooth decline in $\mathrm{O}_{3}$ photolysis rates as the SZA passes from 84 to $95^{\circ}$. Figure $3 \mathrm{~b}$ shows the corresponding 

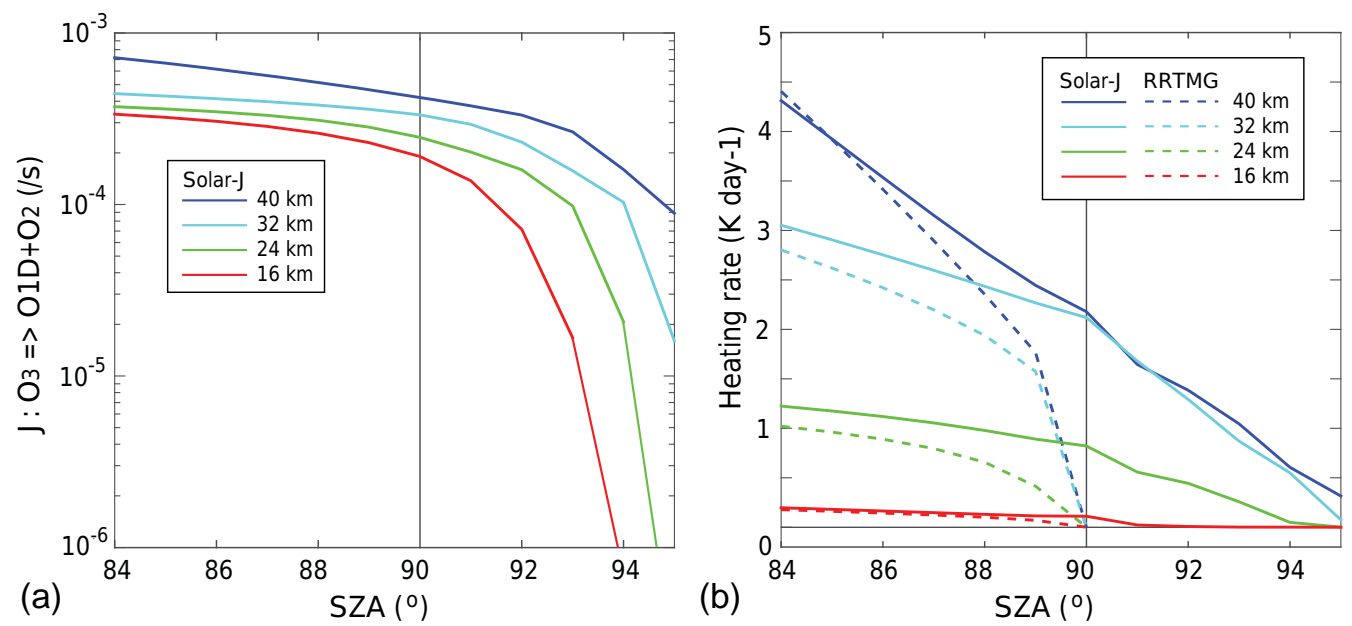

Figure 3. Ozone photolysis rates $\left(J_{\mathrm{O}_{3}}\right)$ from Solar-J (a) and the corresponding atmospheric heating rates under clear sky (b) from Solar-J (solid lines) and RRTMG-SW (dashed lines) for large solar zenith angles at four different altitudes. RRTMG-SW's heating rates reduce to zeros at an SZA of $90^{\circ}$ due to the lack of sphericity correction in the plane-parallel approximation, whereas the impact of sphericity on the direct solar beam path is included in Solar-J.

heating rates from both RRTMG-SW and Solar-J. The lack of sphericity in RRTMG leads to large systematic negative biases in the heating rates at low sun. Sphericity errors extend up to an SZA of $80^{\circ}$ but are largest of course at twilight. The high-latitude atmosphere will have SZAs greater than $80^{\circ}$ for much of the day, and thus RRTMG may lead to a cold bias for the high latitudes.

\subsection{Low-level marine stratus cloud}

For the stratus cloud, the liquid-water path (LWP, $\mathrm{g} \mathrm{m}^{-2}$ ) in each layer is derived from the LWC and height of each layer (Table 3) and is plotted versus altitude in Fig. 4a as described in Sect. 2.3. The resulting cloud optical depth in each layer, $\tau$, (evaluated at $600 \mathrm{~nm}$ ) is also written in pairs with SolarJ's as the first number and RRTMG's reduced delta-scaled optical depth $\left(\tau^{\prime}\right)$ as the second. Both RRTMG and Solar-J start with same value of $\tau$ because the Mie-based scattering phase functions for liquid water are unambiguous and both adopt the same values for $r_{\mathrm{e}}, Q$, and density of liquid water. The $r_{\mathrm{e}}$ is set to $9.6 \mu \mathrm{m}$ through most of this cloud profile. The LWC increases from the surface to a maximum of $0.12 \mathrm{~g} \mathrm{~m}^{-3}$ at $1.25 \mathrm{~km}$ and falls to zero by $2.3 \mathrm{~km}$ altitude. Because of the increasing thickness of the model layers with altitude, the LWP and layer $\tau$ are not as smoothly peaked. We deem this profile realistic from comparing to the observed range for coastal marine low clouds (see Fig. 4 of Hu et al., 2007 for July liquid cloud radii distribution and Fig. 1a of Painemal et al., 2016 for LWP).

Table 5 summarizes the clear-sky radiative budget and the stratus cloud radiative effect $\left(\mathrm{CRE}, \mathrm{W} \mathrm{m}^{-2}\right.$, calculated as change relative to clear sky) for Solar-J and RRTMG-SW for the four SZAs used here. At overhead sun $\left(\mathrm{SZA}=0^{\circ}\right)$ with the solar input at $1360.8 \mathrm{~W} \mathrm{~m}^{-2}$, the effect of this low-level marine stratus cloud (per Solar- $\mathrm{J}$ ) is to reflect an additional $473 \mathrm{~W} \mathrm{~m}^{-2}$ back to space, absorb an additional $88 \mathrm{~W} \mathrm{~m}^{-2}$ in the atmosphere primarily within the cloud, and thus reduce the surface heating from 969 to $408 \mathrm{~W} \mathrm{~m}^{-2}$. As in the clear-sky comparison, both models look broadly similar but with some large systematic biases. For SZAs of 0 $62^{\circ}$, Solar-J reflects $\sim 10 \mathrm{~W} \mathrm{~m}^{-2}(2-3 \%)$ more sunlight back to space, both models calculate about the same increase in atmospheric absorption, and RRTMG-SW consistently absorbs less energy within the cloud but more above it; thus, Solar-J calculates greater reduction in surface heating (also about $2-3 \%$ ) than RRTMG-SW. These differences in solar heating are large compared with anthropogenic climate forcing from greenhouse gases $\left(\sim 4 \mathrm{~W} \mathrm{~m}^{-2}\right)$ (Myhre et al., 2013), but of course stratus clouds occupy only a fraction of the surface. Within the atmosphere, there is a large difference in the distribution of CRE, with Solar-J calculating $5 \%\left(\mathrm{SZA}=0^{\circ}\right)$ to $25 \%\left(\mathrm{SZA}=62^{\circ}\right)$ more in-cloud heating than RRTMG. The profile of heating rates (Fig. $4 \mathrm{~b}$ ) shows a double peak at $1.9 \mathrm{~km}$ (visible $\tau \sim 1)$ and $1.2 \mathrm{~km}(\tau \sim 6)$ even though the LWC has a smooth maximum at $1.1 \mathrm{~km}$. The longer wavelength bins (S25-S27) are fully absorbed in the uppermost part of the cloud $(\tau<1)$, while the shorter wavelengths (S19-S24) penetrate the cloud to scattering optical depths of order $\tau \sim 8$. RRTMG consistently calculates lower in-cloud rates; see below. It is possible that Solar-J's greater heating in stratus clouds may change the dynamics of stratus clouds relative to a model using RRTMG-SW (Harrington et al., 2000). At low sun $\left(\mathrm{SZA}=84^{\circ}\right)$, Solar-J calculates $4 \%$ greater reflectance change, both models calculate less atmospheric heating within the cloud but more heating above it, and the surface heating in Solar-J is about $2 \mathrm{~W} \mathrm{~m}^{-2}$ less than 


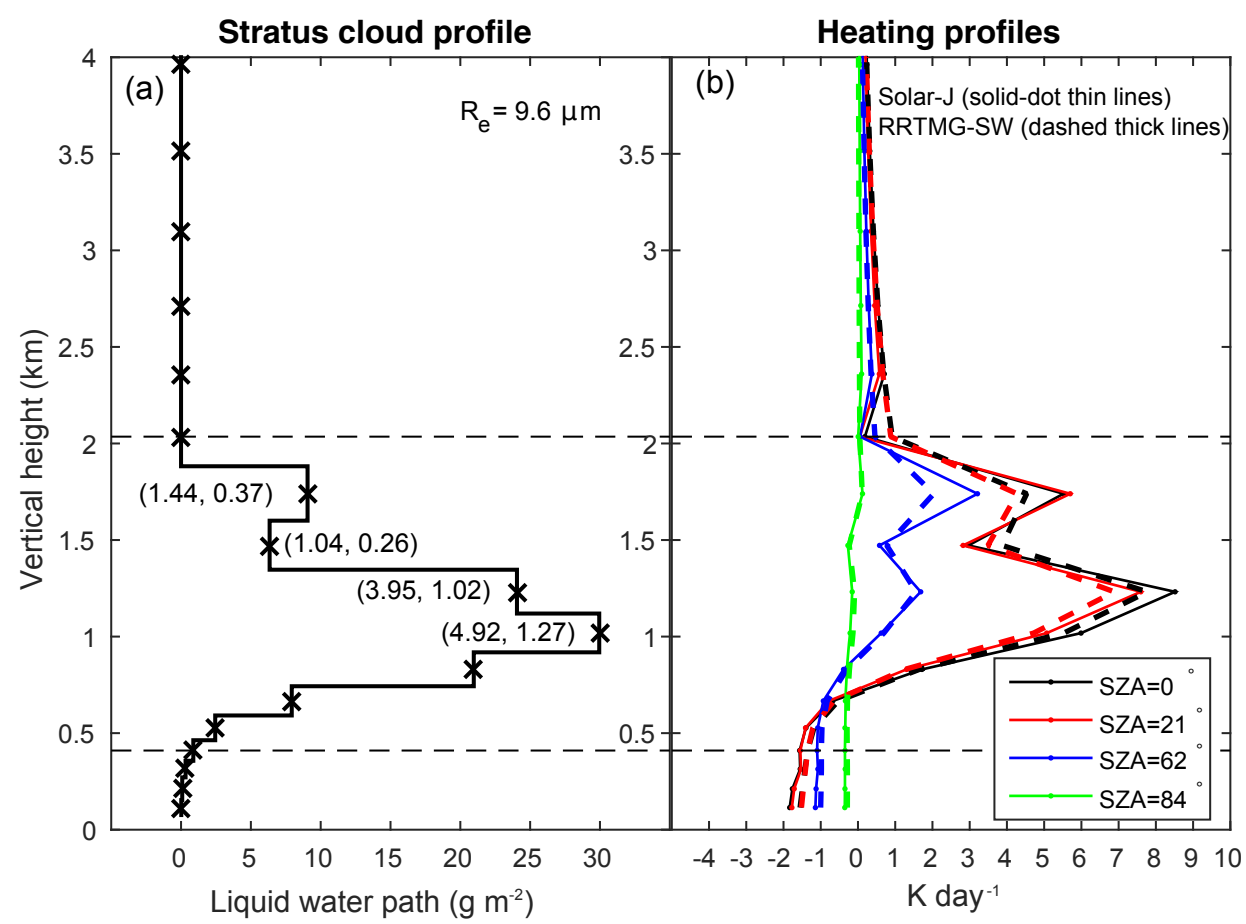

Figure 4. (a) Marine stratus cloud profile in terms of liquid-water path (LWP, $\mathrm{g} \mathrm{m}^{-2}$ ) and effective radius $\left(r_{\mathrm{e}}, \mu \mathrm{m}\right)$. The optical depth and delta-scaled optical depth $\left(\tau, \tau^{\prime}\right)$ are shown in parentheses for the top five cloud layers. (b) Cloud heating profiles from Solar-J (solid lines) and RRTMG-SW (dashed lines) at four SZAs.

in RRTMG-SW. Both models show enhanced heating only in the uppermost cloud layers above $1.7 \mathrm{~km}$ (Fig. 4b).

We believe that the RRTMG-SW biases identified here are errors caused by the two-stream approximation. This is supported by the study of Li et al. (2015; see their Fig. 2), who show small negative errors in absorption from the calculation of delta-Eddington (two-stream) approximation in the case of the single-layer liquid cloud $\left(r_{\mathrm{e}}=10 \mu \mathrm{m}, \tau \sim 4\right)$ with $\cos (\mathrm{SZA})>0.2$ (i.e., our SZAs of $0-62^{\circ}$ ). For our SZA of $84^{\circ}$, this absorption bias reverses as is also found by $\mathrm{Li}$ et al. (2015) for $\cos (\mathrm{SZA})<0.2$. In their study, the twostream calculations are compared to the 128-stream DISORT (discrete-ordinate) benchmark calculations using accurate phase functions and no delta scaling (similar to the study of Wild et al., 2000). One other source of error in RRTMG's model is the choice of delta-scaling factor, which they base on the HG phase function using only $g$. Alternatively, one can use the second moment of the true Mie phase function (Wiscombe, 1977). We revised the RRTMG-SW code to do this using Solar-J's scattering phase functions and found a modest reduction in this error from -14 to $-9 \mathrm{~W} \mathrm{~m}^{-2}$ for reflected sunlight $\left(\mathrm{SZA}=0^{\circ}\right)$.

\subsection{Tropical cirrus clouds}

For the cirrus cloud comparison, we use all three ice-water parameterization options in RRTMG-SW and Solar-J's sin- gle parameterization. Figure 5a, b show the prescribed profiles of model input of IWC and $r_{\mathrm{e}}$ (Table 3 ). The cumulative overhead $\tau$ at $600 \mathrm{~nm}$ is shown in Fig. 5c. The delta scaling varies considerably across the RRTMG parameterizations: Solar-J's unscaled $\tau \sim 0.43$ compares with EC92's $\tau \sim 0.25$, Fu96's $\tau \sim 0.15$, and Key02's $\tau \sim 0.09$ (see also Table 6). Thus, the fraction of sunlight scattered by the cloud varies widely across all four. The asymmetry parameter $g$ from Mishchenko's phase functions for hexagonal and irregular ice used in Solar-J ranges from 0.75 to 0.81 (as compared to 0.88 for equivalent-size liquid-water clouds), but $g$ values for all RRTMG ice clouds range from 0.4 to 0.6 for wavelengths where scattering is important (S12-S24). The absorbing optical depth, $\tau_{\text {abs }}$, is a very important diagnostic because in an optically thin cloud the overall heating should be proportional to it. Table 6 shows that all four ice cloud models have similar $\tau_{\text {abs }}$ up to S22, and if we average S23 and S24 (which appears to have been done in EC92), then all four models remain similar in terms of solar absorption. As noted for the stratus cloud, all models predict a large, factor of 5, jump in $\tau_{\mathrm{abs}}$ for S25-S27 $(\lambda>2.5 \mu \mathrm{m})$, which are the most important bins for cirrus cloud heating. At these wavelengths, EC92 has the largest absorption $\tau_{\text {abs }}$, about 0.3 , followed by Solar-J's 0.21.

Cloud heating rate profiles at an SZA of $0^{\circ}$ are shown in Fig. 5d, and the large range clearly reflects the $\tau_{\mathrm{abs}}$ for S25-S27. The cirrus CRE for four SZAs and for five com- 


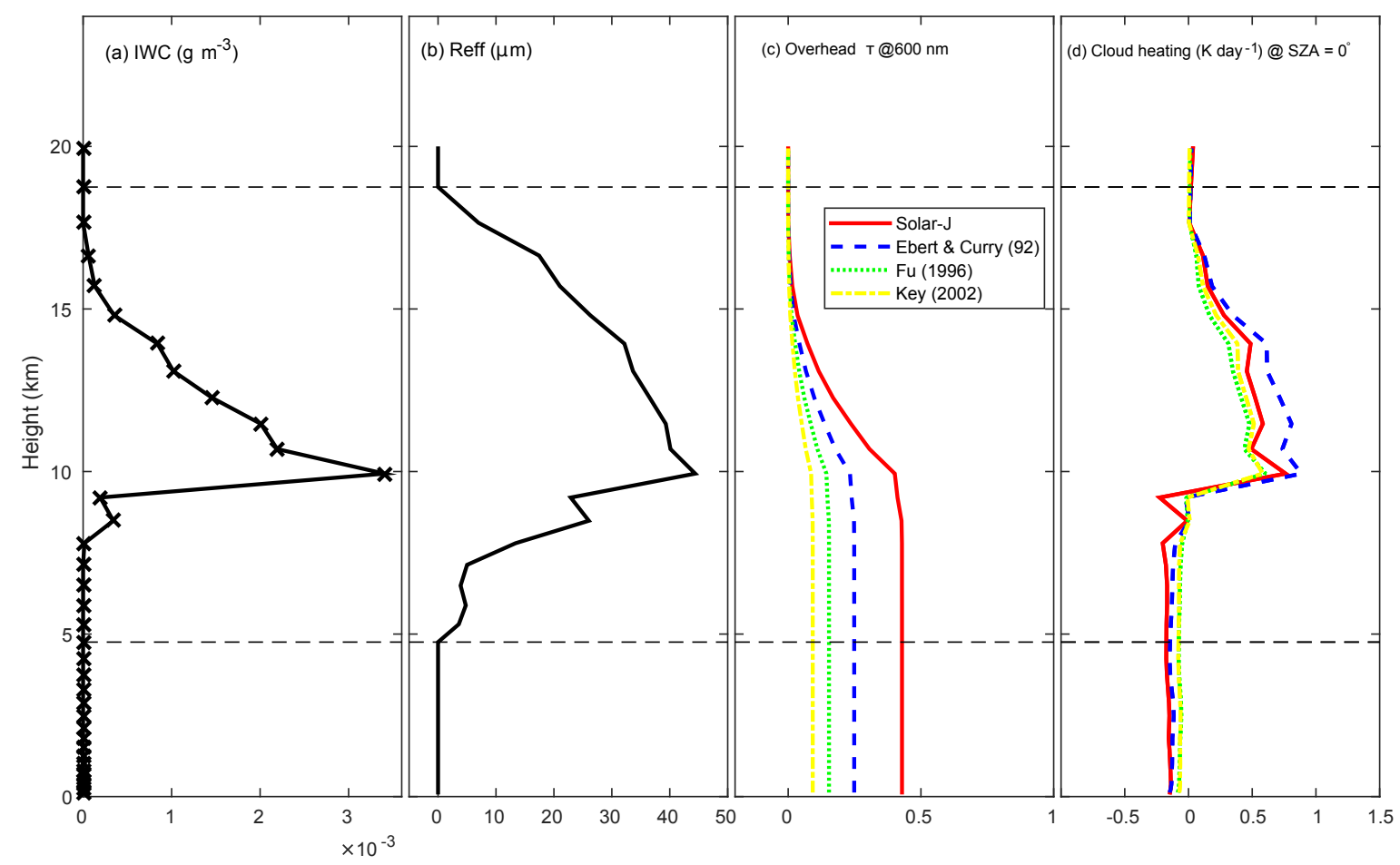

Figure 5. (a) Profiles of ice-water content (IWC, $\mathrm{g} \mathrm{m}^{-3}$ ) and (b) effective radius $\left(r_{\mathrm{e}}, \mu \mathrm{m}\right)$ as prescribed for both Solar-J and RRTMG-SW. The in-cloud region, about $4-18 \mathrm{~km}$, is enclosed by two horizontal dashed lines. (c) Profiles of cumulative optical depth $\tau$ at $600 \mathrm{~nm}$ from Solar-J and from the three RRTMG-SW parameterizations for which $\tau$ is delta scaled. (d) Cirrus cloud heating rate profiles (K day ${ }^{-1}$ ) at an SZA of $0^{\circ}$.

ponents (reflected at top of atmosphere, absorbed in abovecloud atmosphere, in-cloud atmosphere, below-cloud atmosphere, and absorbed at the surface) are shown as a set of 20 bar charts in Fig. 6. The CRE percent changes relative to clear sky are shown as four color bars representing Solar-J (red), EC92 (blue), Fu96 (green), and Key02 (yellow). The clear-sky energy flux $\left(\mathrm{W} \mathrm{m}^{-2}\right)$ averaged over the four models is shown in a larger font in each bar chart. For example, at an SZA of $21^{\circ}$ the energy absorbed by clear-sky atmosphere over the altitude range of the cirrus cloud is $98.4 \mathrm{~W} \mathrm{~m}^{-2}$. The $\mathrm{CRE}$ in $\mathrm{W} \mathrm{m}^{-2}$ within the cirrus cloud for Solar-J is then $98.4 \times 10.7 \%$ (red bar) $=+10.5$. The $y$ axes in Fig. 6 have different scales at different SZAs.

A key cirrus CRE is the increase in albedo, the top-ofatmosphere reflected sunlight, as shown for all models and a range of SZAs in Fig. 6 (top row). The percent increase across RRTMG-SW models (13-131\%) scales in proportion to $\tau$, with EC92 being the largest and Key02, the smallest. This relative order stays the same across all SZAs, but the range across RRTMG-SW models decreases and the relative percent increases for larger SZAs. The Solar-J model also increases in percent with SZA, but the pattern is different from that of RRTMG-SW models. At overhead sun, Solar-J has about the same CRE percent as EC92 even though it has a 1.7 times greater $\tau$. This can be understood in that Solar-
$\mathrm{J}$ cirrus is highly forward scattering and less of the scattered light is reflected backward and upward. As the SZA increases to $21-62^{\circ}$, however, the peak in backscatter at $180^{\circ}$ becomes less important and Solar-J shifts lower relative to EC92 to look like Fu96. At very large SZA of $84^{\circ}$, with most of the sunlight being scattered at least once within the cloud, the Solar-J model again looks like the largest $\tau$, model EC92. To first order, the Solar-J model is calculating the correct SZA dependence of the CRE by using both a more realistic scattering phase function and eight-stream scattering. The use of Mishchenko's sample T-matrix phase function may not be a perfect choice for cirrus, but it is clearly more realistic than the isotropic scattering used in RRTMG-SW. Solar-J captures the cirrus albedo curve similar to Fig. 2 of Mishchenko et al. (1996) for $\tau=0.1$ in which the slope increases rapidly as the cosine (SZA) approaches 0. While the RRTMG-SW two-stream models can be tuned to be correct at some SZAs, they will have errors of $15 \mathrm{~W} \mathrm{~m}^{-2}$ at others. The change in surface heating (fifth row) looks like the reverse of the top-of-atmosphere bars with similar relative weighting of the RRTMG-SW models. Again, it shows that two-stream scattering cannot mimic the correct SZA dependence of reduced surface heating under cirrus.

With greater reflection of sunlight, the atmospheric heating above the cloud increases in all cases. With RRTMG-SW, 

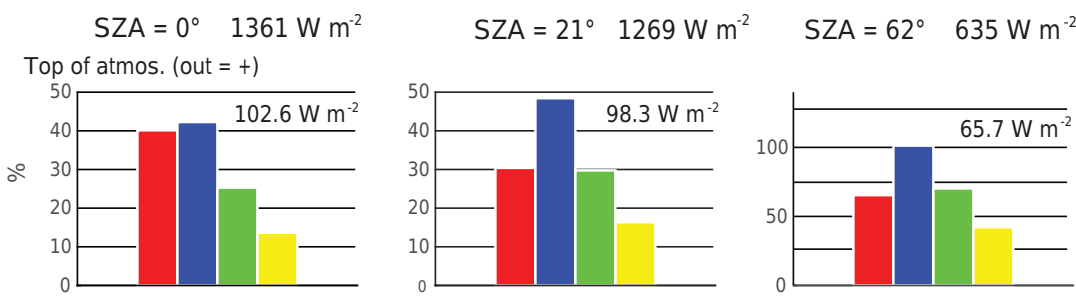

$\mathrm{SZA}=84^{\circ} \quad 149 \mathrm{~W} \mathrm{~m}^{-2}$

Above-cloud atmos. (abs $=+$ )
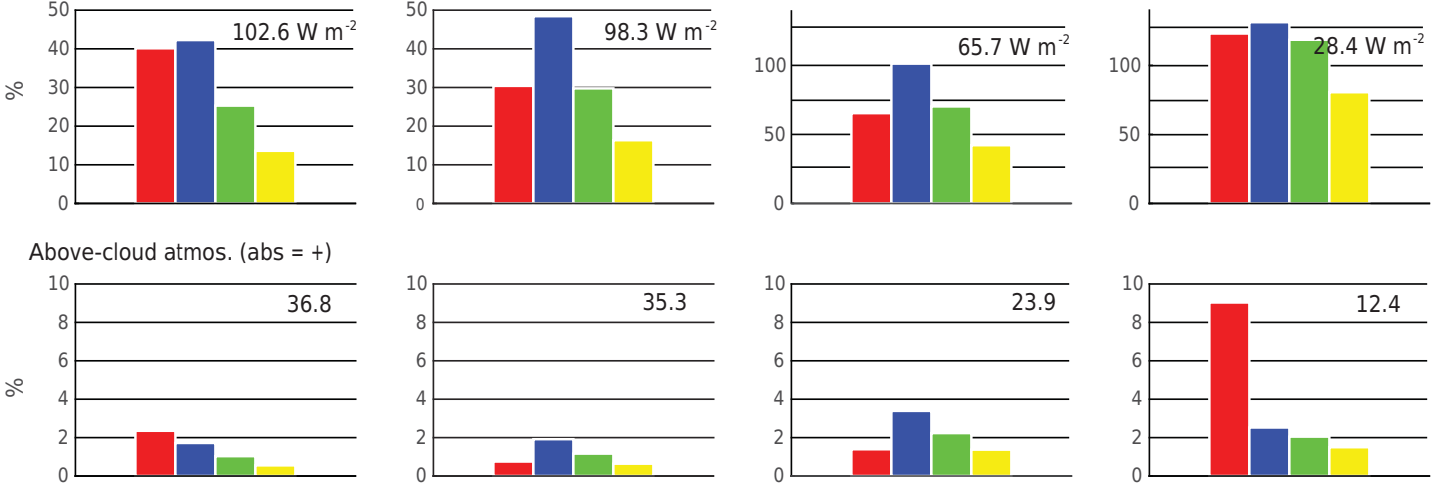

In-cloud atmos. \& cloud (abs $=+$ )
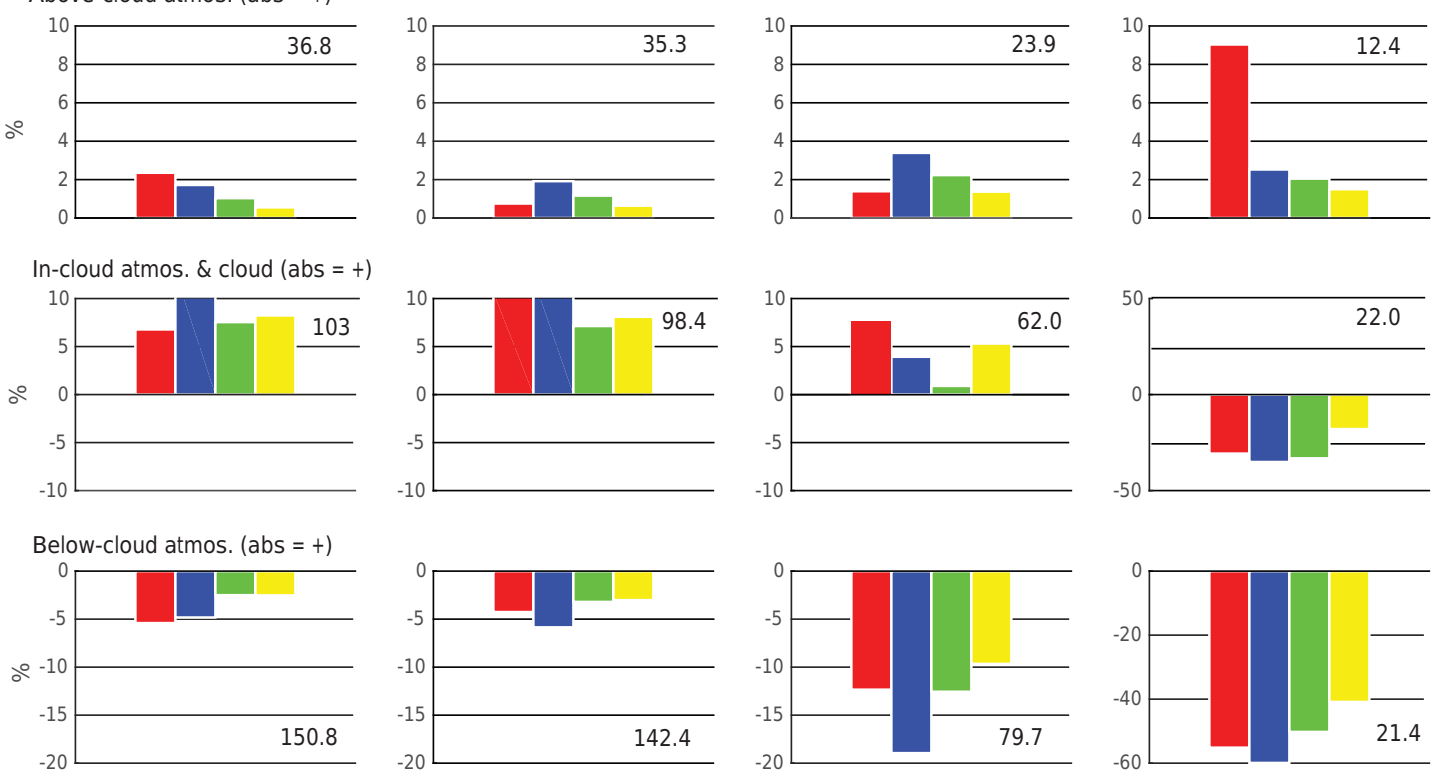

Surface $(\ln =+)$
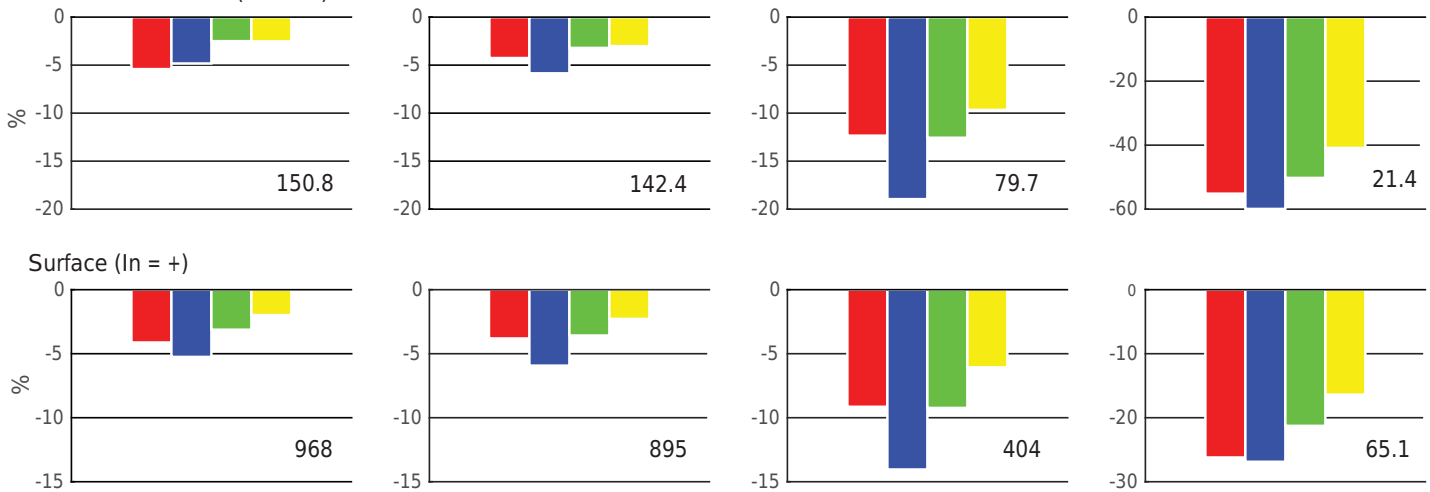

Cirrus model:

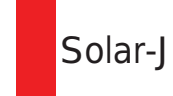

$\mathrm{E} \& C 92$

Fu 96

Key 02

Figure 6. Percent changes (\%) in shortwave radiation energy budget relative to the aerosol-free clear sky (surface albedo of 0.06 ) caused by a cirrus cloud using four different models: Solar-J and the three RRTMG-SW parameterizations for ice clouds. Results are shown for four different solar zenith angles. Changes in the vertical column are divided into five regions: top of atmosphere, atmospheres above, within, and below the cirrus cloud, and at the surface. Single numbers in bold shown in the corner of each panel are the clear-sky energy budget in $\mathrm{W} \mathrm{m}^{-2}$ averaged over Solar-J and RRTMG-SW for each region. Note that different $y$-axis scales have been used for large SZAs.

the scattered light has only one angle, and thus the abovecloud heating (second row of Fig. 6) is strictly proportional to the top-of-atmosphere increases. With Solar-J, the reflected light is calculated at four zenith angles with the flux at larger zenith angles producing more heating (i.e., longer slant path through the atmosphere). This is most apparent in the case of the SZA of $84^{\circ}$ where the low-angle scattering driven by the low solar elevation produces relatively much more atmospheric heating.

In-cloud heating (third row) is expected to be proportional to $\tau_{\text {abs }}$ at high sun $\left(\mathrm{SZA}=0-62^{\circ}\right)$, and for flux-weighted bins S25-S27 these $\tau_{\text {abs }}$ are 0.31 (EC92), 0.21 (Solar-J), 0.17 (Key02), and 0.16 (Fu96). While the actual heating of the cirrus ice particles may be in similar proportionality, all we calculate is the total change of heating over the in-cloud layers. As seen in Fig. 6, there is substantial clear-sky absorption by atmospheric water vapor in the cloudy layers $\left(\sim 100 \mathrm{~W} \mathrm{~m}^{-2}\right)$ at high sun. Thus, the small perturbation caused by the cloud $(<10 \%$, third row) results from in-cloud heating of ice particles (proportional to $\tau_{\mathrm{abs}}$ ) countered by reduced heating of the water vapor in the region because of the increased upward scattered light (top row). The extreme case of the SZA of $84^{\circ}$ 
has all models calculating $20-35 \%$ reductions in heating because of the reduced sunlight.

\section{Computational costs}

The major computational costs of Solar-J and similar codes within a chemistry-climate model centers on three key components: matrix operations required for multi-stream scattering, wavelength bins representing the spectrum of optical properties, and approximation of the multitude of independent column atmospheres (ICAs) resulting from a complex overlapping cloud field within a grid cell. What is a reasonable requirement for multi-stream scattering in a climate model? From this work as well as a history of publications noted above, the analytic two-stream approximation has errors that cannot simply be corrected or averaged over, that create large-scale biases in cloud radiative forcing with latitude, and that significantly misrepresent the direct-diffuse ratio of solar radiation at the surface. The original Fast-J work (Wild et al., 2000) examined a range of multi-stream scattering models and found that for typical clouds, an eight-stream solution was able to match within a few percent that of a 100-stream code for the mean intensity above, within and below the cloud. A major advantage of the eight-stream system was that no delta scaling was needed and a simply truncated scattering phase function could be used directly. The parent RRTM-SW code has the option of using a more accurate 16-stream scattering code but would in general be computationally much more expensive than the eight-stream Solar-J. The basic costs of the matrix inversions (Fast-J via Feautrier, 1964) or eigenvalue solutions (RRTM via DISORT; Stamnes et al., 1988) scale as $n^{3}$. For the same eight-stream solution, DISORT performs eigenvalue decomposition of $8 \times 8 \mathrm{ma}-$ trices at each level at a cost of order $8^{3}$, while the Feautrier approach solves the finite-difference equations with $4 \times 4$ matrices at split levels for a cost of order $2 \times 4^{3}$. As a first guess, the Feautrier code should run 4 times faster than the equivalent DISORT code. We examine the costs and options of wavelength binning and cloud-field approximations below.

\subsection{Solar-J versus RRMTG-SW}

Cloud-J (and hence Fast-J) has been extensively tested in the UC Irvine chemistry-transport model (CTM). Cloud-J timings are estimated by comparing full cloud quadrature (2.75 calls per column atmosphere per time step; see below) versus an average-cloud approximation ( 1 call). We find that $12 \%$ of the CTM wall-clock time is spent in Cloud-J using average clouds and $28 \%$ when using cloud quadrature. Because the UCI CTM runs a minimalist tropospheric chemistry and a linearized stratospheric chemistry (see Hsu and Prather, 2010), it keeps track of only 32 species. More complete models like Oslo CTM3 (Søvde et al., 2012) and WACCM (Marsh et al., 2013) calculate transport and chem- istry of about 100 species. In CTMs like these, the fractional cost of Cloud-J should be only 4-7\%. Comparing Solar-J to Cloud-J in single-atmosphere tests shows what is expected; Solar-J costs are 3.5 times greater because of the much larger number of spectral bands needed for heating (100 versus 18). A minor feature is that cloudy atmospheres cost about $10 \%$ more than clear atmospheres because Cloud-J inserts extra layers at the top of clouds to enhance the accuracy of the finite-difference equations.

In a series of comparisons on a single-socket multithreaded CPU, we find that Solar-J takes 5 times more wall clock time than RRTMG-SW. This is not surprising given the cost of solving an eight-stream versus two-stream RT problem. An additional cost of Solar-J (not included above) is spherical geometry. With RRTMG-SW, $50 \%$ of the grid cells are in sunlight and require RT solutions. With Solar-J, however, important photochemistry and solar heating occur in the atmosphere when the surface is past sunset (see Fig. 3) involving about $56 \%$ of the grid cells, a $12 \%$ increase in radiatively active grid cells. One could expect that RRTMGSW will correct this error and end up with similar increases in coverage and cost.

Most climate models, even at the highest resolutions, have individual grid cells with fractional, overlapping cloud layers. Although 3-D RT models can be used to solve for the average heating and photolysis rates, most climate models decompose the cloud structures into ICAs, for example, through cloud-resolving models (Khairoutdinov et al., 2005) or from cloud fractional coverage and a decorrelation distance for overlapping cloud layers (Prather, 2015). The ICAs are horizontally homogeneous and can be solved using the 1-D RT codes of RRTMG-SW or Solar-J. Comparisons between Solar-J and RRTMG-SW for clouds in Sect. 3 are done with a single 1-D plane-parallel, ICA-like atmosphere (i.e., $100 \%$ cloud fraction in each cloud layer), an idealized case.

Although the different approaches for fractional cloud cover were not directly tested here, it is worth looking at how Solar-J and RRTMG might treat cloud fields in climate models. The Monte Carlo ICA (McICA; Pincus et al., 2003) method selects both ICAs and spectral intervals randomly in each grid square. Every spectral interval is sampled only once, and each may have a different ICA selected according to its fractional area (frequency of occurrence). With $100+$ bin-ICA combinations, the ICAs are well sampled, but there may be instances in which a few key large-energy bins are not sampled accurately. The McICA approach, when suitably averaged over time, has no mean bias in average heating rates but very large root mean square (rms) errors: e.g., $\pm 105 \mathrm{~W} \mathrm{~m}^{-2}$ in surface heating with an SZA of $45^{\circ}$; $\pm 3 \mathrm{~K} \mathrm{day}^{-1}$ in layers with partly cloudy atmospheres (Pincus et al., 2003). It is cost efficient in that each wavelength bin requires only one ICA calculation. Solar-J uses cloud quadrature, introduced by Neu et al. (2007), selecting up to four cloud profiles (QCAs) based on total optical depth to represent four types of atmospheres: mostly clear, typical cir- 
rus clouds, typical stratus clouds, and very thick frontal or cumulus clouds. While each grid cell may have up to four QCAs, on average there are only 2.75 . Solar-J then calculates all wavelength bins using all QCAs to compute the average. Cloud-J (Prather 2015) compared several approximations for calculating average photolysis rates (Js) within a sample of 640 tropical atmospheres where the generated number of ICAs per grid cell ranged from 1 to 3500 and averaged 170 . Compared to the exact answer defined by separate calculations with all the weighted ICAs, cloud quadrature achieves rms errors in instantaneous cell-averaged Js of 0 to $3 \%$ throughout the troposphere, with most levels being $0-1 \%$. When Cloud-J is run selecting random ICAs (using all wavelengths for each ICA, not the McICA approach), 50 random ICAs (18 times greater cost) are needed to achieve the accuracy of cloud quadrature.

From the point of view of chemistry-climate models, large rms errors in Js cannot be tolerated because the chemistry is non-linear and such errors are not likely to average. In climate models, there are threshold processes like aerosol and ozone heating preventing cloud formation (e.g., Koch and DelGenio, 2010), for which heating noise may not simply average out. Errors in heating rates do not always have symmetric responses in terms of climate (e.g., Hsu et al., 2013). Although Pincus et al. (2003) tested climate forecasting with an early version of McICA, it is not clear how forecast skill with modern, high-resolution models is impacted by the biases in RRTMG-SW. Alternatively, RRTMG-SW could adopt cloud quadrature with 2.8 times greater cost and eliminate most of their rms errors in heating.

All of these standard features of Solar-J (eight streams, spherical geometry, cloud quadrature) increase the computational cost, but one can argue that the improved fidelity in the solar heating of the atmosphere and radiative forcing of the climate is worth the cost. The question is what fraction of the total computational cost of a climate simulation would be used by Solar-J. If we estimate the fractional cost of RRTMG in a full atmosphere-ocean climate simulation to be $1-3 \%$, then replacing it with Solar-J (5 times greater) and including cloud quadrature (2.8 times greater), would increase this to $13-39 \%$. At the lower end of this range, the substantially improved and less noisy physics is probably worth it, but at the upper end it is prohibitive. In either case, it is worthwhile to pursue a range of computer science and algorithmic approaches to reduce these costs as discussed in Sect. 4.2 and 4.3 below.

\subsection{Computer science options}

A profiling of the Solar-J code shows that the Fast-J core, consisting of a scattering matrix generator and blocktridiagonal solver, is the dominant cost. These two subroutines are already well optimized in terms of single CPU multi-threading; however, porting Fast-J to computers with graphical processing units (GPUs) has shown promise for greater speed. One effort targeted a single GPU and demonstrated speedups via CUDA (Compute Unified Device Architecture) tuning of $\sim 50$ times more relative to the $\mathrm{CPU}$ time if a large number of column atmospheres $(200+)$ were concurrently evaluated (Artico et al., 2015). Another effort used a field-programmable gate array (FPGA) with the advantage that it applies to a single column calculation. The FPGA resulted in a $\sim 4$ times greater speedup and a rather dramatic 35 times more energy savings compared to the multicore processor computation (Rezaei et al., 2016). Fast-J was also optimized for the Xeon Phi on the Babbage test platform at DOE National Energy Research Scientific Computing Center (NERSC) and achieved $\sim 3$ times faster speedups with only a subset of the cores.

Great computational acceleration could be realized with GPU systems when a number of column atmospheres are being simultaneously evaluated. For each grid cell, Solar-J calculates about 100 wavelength bins and an average of 2.75 ICAs per grid square. Giving each CPU/GPU node a $3 \times 3$ grid cell square $(\sim 2500$ column atmospheres) could achieve 10 times greater or faster speedups for Solar-J and be appropriate for a massively parallel climate simulation (e.g., 32000 nodes for a $50 \mathrm{~km}$ global grid). With such speedups, Solar-J costs would be comparable or possibly less than those of the current RRTMG-SW and thus become a marginal cost in the climate simulation.

\subsection{Other parameterizations for wavelength bins}

Solar-J uses its own optimization of wavelength bins at ultraviolet and visible wavelengths $(0.18$ to $0.8 \mu \mathrm{m})$, which is based on previously established experience with $\mathrm{O}_{2}$ and $\mathrm{O}_{3}$ cross sections and the need to calculate accurate $\mathrm{J}$ values. We accept that RRTMG-SW and its parent code RRTM represent current best practice and accuracy in characterizing the absorption of infrared sunlight $(0.8$ to $12 \mu \mathrm{m})$ in the Earth's atmosphere and have adopted the RRTMG-SW code exactly for bins and all gaseous absorbers. Solar-J's computational cost is clearly driven by the additional 82 infrared bins adopted from RRTMG-SW. Alternative methods of parameterizing these infrared bins need to be examined: e.g., 14 bins (Chou, 1992; Grant and Grossman, 1998), 34 bins (Fu and Liou, 1992), and 36 bins (Cole, 2005). Any of these would result in 1.5-2.5 times more savings for Solar-J. We recognize that the infrared bins adopted in RRTMG-SW are based on accurate representation of the line-by-line calculations, and thus adopting these reduced-bin parameterizations will introduce new errors, but further research will be needed to determine whether these errors maybe an acceptable tradeoff for speed gain.

Many of these other parameterizations (e.g., Chou, 1992) are based only on water vapor and do not include the other trace gases that are represented in RRTMG-SW: $\mathrm{O}_{2}$ in the visible and infrared, $\mathrm{CH}_{4}$, and $\mathrm{CO}_{2}$. These gases add to the complexity of the RRTM-SW model, and thus we investigate 
their importance in tropospheric heating rates. For our clearsky case here (Table 4, Fig. 2), we find an average tropospheric heating rate of $2.4 \mathrm{~K} \mathrm{day}^{-1}$. The contribution of $\mathrm{CH}_{4}$ to this total is $0.1 \%$; that of $\mathrm{CO}_{2}$ is complex because of the stratospheric self-shielding but is less than $\pm 1 \%$ in the troposphere, and that of $\mathrm{O}_{2}$ is about $3 \%$ uniformly throughout the troposphere. If we can find a way of treating the $\mathrm{O}_{2}$ heating separately, then the effort to find an abbreviated number of spectral intervals can focus on water vapor.

\section{Conclusions}

We present a new solar radiation module. Solar-J version 7.5 aims for consistent calculation of atmospheric photolysis and solar heating rates by combining the strengths of both CloudJ and RRTMG-SW. In a chemistry-climate model, Solar-J supplies the needs of solar heating of the atmosphere and surface, photolysis rates, and photosynthetic activity. Climate models are increasingly including short-lived gases and aerosols as radiative forcing components, and the accurate simulation of these under different climates requires some level of interactive chemistry and photolysis rates.

The components of Solar-J are chosen and balanced to achieve the best possible overall accuracy for a module intended as a standard component of chemistry-climate simulations. From Cloud-J, we take the eight-stream scattering model, semi-spherical geometry, ultraviolet transmission, and cloud quadrature. From RRTMG-SW, we take the detailed spectral intervals for the visible and infrared developed from the RRTM reference code. Solar-J matches RRTMGSW except where the improved physics leads to more accurate results. Selecting the best physics for all these components comes with a cost: a simple comparison shows the cost of Solar-J is 5 times that of RRTMG-SW for a single atmosphere, and if the cloud quadrature scheme for overlapping cloud fields (Neu et al., 2007; Prather, 2015) is applied to either code, the cost increases additionally by 2.8 times. We show that Solar-J can be optimized on GPUs and achieve speeds similar to RRTMG-SW. While this opens up great opportunities for the new generation of high-performance computers, it also complicates the simple implementation of Solar-J in a climate model.

Solar-J is a starting point. In assessing the fidelity of Solar$\mathrm{J}$ in terms of interaction with the many components of the climate system, we can focus on the three major sources of costs/error (spectral intervals, multi-stream radiative transfer, and complex cloud systems) and, in parallel, on the opportunities for accelerated performance with new computational architectures. Ideally, the community would optimize computational costs across modules to have comparable (small) errors in all. In addition, these parameterization errors need to be evaluated for the impact they might have on climate simulations (e.g., Pincus et al., 2003). For Solar-J, the next steps consist of (i) moving the S17-S18 boundary to the be- ginning of the $\mathrm{O}_{3}$ Chappuis absorption near $0.5 \mu \mathrm{m}$, and (ii) developing a more realistic and diverse range of cirrus clouds and their optical properties (e.g., Yang et al., 2015). Another inquiry is to test some of the published, simpler models for water vapor absorption against RRTMG-SW. A larger project will be to put Solar-J into a climate model and evaluate how errors in modeling solar radiation may affect the climate simulations.

Code availability. The most recent version of Solar-J can be found at ftp://128.200.14.8/public/junoh/Solar-J/. A complete version of Solar-J code and data, along with some stand-alone test cases, is included in a zip file as a Supplement to this article.

\section{The Supplement related to this article is available online at https://doi.org/10.5194/gmd-10-2525-2017- supplement.}

Competing interests. The authors declare that they have no conflict of interest.

Acknowledgements. This work is supported by the US Department of Energy, Office of Science, Biological and Environmental Research Program under award nos. DE-SC0007021 and DESC0012536, and by NASA Modeling, Analysis and Prediction (MAP) program under award no. NNX13AL12G. This research used resources of the National Energy Research Scientific Computing Center, a DOE Office of Science User Facility supported by the Office of Science of the US Department of Energy under contract no. DE-AC02-05CH11231. Part of this work was performed under the auspices of the US Department of Energy by Lawrence Livermore National Laboratory under contract no. DE-AC52-07NA27344.

Edited by: Gerd A. Folberth

Reviewed by: two anonymous referees

\section{References}

Artico, F., Prather, M. J., Veidenbaum, A. V., and Nicolau, A.: Fast Fast-J GPU Codes, CECS technical report, 15-03, 8 November 2015.

Barker, H. W., Cole, J. N. S., Li, J., Yi, B., and Yang, P.: Estimation of Errors in Two-Stream Approximations of the Solar Radiative Transfer Equation for Cloudy-Sky Conditions, J. Atmos. Sci., 72, 4053-4074, https://doi.org/10.1175/JAS-D-15-0033.1, 2015.

Beyer, K. D., Ravishankara, A. R., and Lovejoy, E. R.: Measurements of UV refractive indices and densities of $\mathrm{H}_{2} \mathrm{SO}_{4} / \mathrm{H}_{2} \mathrm{O}$ and $\mathrm{H}_{2} \mathrm{SO}_{4} / \mathrm{HNO}_{3} / \mathrm{H}_{2} \mathrm{O}$ solutions, J. Geophys. Res.-Atmos., 101, 14519-14524, https://doi.org/10.1029/96JD00937, 1996.

Bian, H. and Prather, M. J.: Fast-J2: Accurate Simulation of Stratospheric Photolysis in Global Chemical Models, J. Atmos. Chem., 41, 281-296, https://doi.org/10.1023/A:1014980619462, 2002. 
Biermann, U. M., Luo, B. P., and Peter, T.: Absorption Spectra and Optical Constants of Binary and Ternary Solutions of $\mathrm{H}_{2} \mathrm{SO}_{4}, \mathrm{HNO}_{3}$, and $\mathrm{H}_{2} \mathrm{O}$ in the Mid Infrared at Atmospheric Temperatures, J. Phys. Chem. A, 104, 783-793, https://doi.org/10.1021/jp992349i, 2000.

Boers, R., Jensen, J. B., and Krummel, P. B.: Microphysical and short-wave radiative structure of stratocumulus clouds over the Southern Ocean: Summer results and seasonal differences, Q. J. Roy. Meteor. Soc., 124, 151-168, https://doi.org/10.1002/qj.49712454507, 1998.

Boucher, O., Schwartz, S. E., Ackerman, T. P., Anderson, T. L., Bergstrom, B., Bonnel, B., Chýlek, P., Dahlback, A., Fouquart, Y., Fu, Q., Halthore, R. N., Haywood, J. M., Iversen, T., Kato, S., Kinne, S., Kirkevåg, A., Knapp, K. R., Lacis, A., Laszlo, I., Mishchenko, M. I., Nemesure, S., Ramaswamy, V., Roberts, D. L., Russell, P., Schlesinger, M. E., Stephens, G. L., Wagener, R., Wang, M., Wong, J., and Yang F.: Intercomparison of models representing direct shortwave radiative forcing by sulfate aerosols, J. Geophys. Res., 103, 16979-16998, https://doi.org/10.1029/98JD00997, 1998.

Boucher, O., Randall, D., Artaxo, P., Bretherton, C., Feingold, G., Forster, P., Kerminen, V.-M., Kondo, Y., Liao, H., Lohmann, U., Rasch, P., Satheesh, S., Sherwood, S., Stevens, B., and Zhang, X.: Clouds and Aerosols, in: Climate Change 2013: The Physical Science Basis. Contribution of Working Group Ito the Fifth Assessment Report of the Intergovernmental Panel on Climate Change, Cambridge University Press, Cambridge, United Kingdom and New York, NY, USA, https://doi.org/10.1017/CBO9781107415324.016, 2013.

Chou, M.-D.: A Solar Radiation Model for Use in Climate Studies, J. Atmos. Sci., 49, 762-772, https://doi.org/10.1175/15200469(1992)049<0762:ASRMFU>2.0.CO;2, 1992.

Clough, S., Shephard, M., Mlawer, E., Delamere, J., Iacono, M., Cady-Pereira, K., Boukabara, S., and Brown, P.: Atmospheric radiative transfer modeling: a summary of the AER codes, J. Quant. Spectrosc. Ra., 91, 233-244, https://doi.org/10.1016/j.jqsrt.2004.05.058, 2005.

Cole, J. N. S.: Assessing the importance of unresolved cloudradiation interactions in atmospheric global climate models using the multiscale modelling framework, $\mathrm{PhD}$ thesis, Pa. State Univ., University Park, 2005.

Deirmendjian, D.: Electromagnetic Scattering on Spherical Polydispersions, American Elsevier, New York, 1969.

Deshler, T., Hervig, M. E., Hofmann, D. J., Rosen, J. M., and Liley, J. B.: Thirty years of in situ stratospheric aerosol size distribution measurements from Laramie, Wyoming $\left(41^{\circ} \mathrm{N}\right)$, using balloon-borne instruments, J. Geophys. Res.-Atmos., 108, 4167, https://doi.org/10.1029/2002JD002514, 2003.

Downing, H. D. and Williams, D.: Optical constants of water in the infrared, J. Geophys. Res., 80, 1656-1661, https://doi.org/10.1029/JC080i012p01656, 1975.

Ebert, E. E. and Curry, J. A.: A parameterization of ice cloud optical properties for climate models, J. Geophys. Res.-Atmos., 97, 3831-3836, https://doi.org/10.1029/91JD02472, 1992.?

Eyring, V., Shepherd, T. G., and Waugh, D. W. (Eds.): SPARC CCMVal Report on the Evaluation of Chemistry-Climate Models, No. 5, WCRP-30/2010, WMO/TD-No. 40, SPARC Office, available at: http://www.sparc-climate.org/publications/ sparc-reports/ (last Access: 26 June 2017), 2010.
Fang, T. M., Wofsy, S. C., and Dalgarno, A.: Opacity Distribution Functions and Absorption in Schumann-Runge Bands of Molecular-Oxygen, Planet. Space Sci., 22, 413-425, 1974.

Feautrier, P.: Sur la résolution numérique de l'équation de transfert, Comptes Rendus Acad. Sci. Paris, 258, 3189, 1964.

Fu, Q.: An Accurate Parameterization of the Solar Radiative Properties of Cirrus Clouds for Climate Models, J. Climate, 9, 2058- 2082, https://doi.org/10.1175/15200442(1996)009<2058:AAPOTS>2.0.CO;2, 1996.

$\mathrm{Fu}, \mathrm{Q}$. and Liou, K. N.: On the correlated k-distribution method for radiative transfer in nonhomogeneous atmospheres, J. Atmos. Sci., 49, 2139-2156, 1992.

Gerber, H.: Microphysics of Marine Stratocumulus Clouds with Two Drizzle Modes, J. Atmos. Sci., 53, 1649-1662, https://doi.org/10.1175/1520 0469(1996)053<1649:MOMSCW>2.0.CO;2, 1996.

Grant, K. E. and Grossman, A. S.: Description of a Solar Radiative Transfer Model for Use in LLNL Climate and Atmospheric Chemistry Studies, UCRL-ID(129949), 17 pp., 1998.

Hale, G. M. and Querry, M. R.: Optical Constants of Water in the 200-nm to 200- $\mu \mathrm{m}$ Wavelength Region, Appl. Optics, 12, 555563, https://doi.org/10.1364/AO.12.000555, 1973.

Hansen, J. E. and Travis, L. D.: Light scattering in planetary atmospheres, Space Sci. Rev., 16, 527-610, https://doi.org/10.1007/BF00168069, 1974.

Harrington, J. Y., Feingold, G., and Cotton, W. R.: Radiative impacts on the growth of a population of drops within simulated summertime Arctic stratus, J. Atmos. Sci., 57, 766-785, https://doi.org/10.1175/15200469(2000)057<0766:Riotgo>2.0.Co;2, 2000.

Henyey, L. G. and Greenstein, J. L.: Diffuse Light in the Galaxy, Astrophys. J., 93, 70-83, https://doi.org/10.1086/144246, 1941.

Hess, M., Koepke, P., and Schult, I.: Optical Properties of Aerosols and Clouds: The Software Package OPAC, B. Am. Meteorol. Soc., 79, 831-844, https://doi.org/10.1175/15200477(1998)079<0831:OPOAAC>2.0.CO;2, 1998.

Heymsfield, A. J., Matrosov, S., and Baum, B.: Ice Water Path-Optical Depth Relationships for Cirrus and Deep Stratiform Ice Cloud Layers, J. Appl. Meteorol., 42, 1369-1390, https://doi.org/10.1175/15200450(2003)042<1369:IWPDRF>2.0.CO;2, 2003.

Hsu, J. N., Prather, M. J., Bergmann, D., and Cameron-Smith, P.: Sensitivity of stratospheric dynamics to uncertainty in $\mathrm{O}_{3}$ production, J. Geophys. Res.-Atmos., 118, 8984-8999, https://doi.org/10.1002/Jgrd.50689, 2013.

Hu, Y., Vaughan, M., McClain, C., Behrenfeld, M., Maring, H., Anderson, D., Sun-Mack, S., Flittner, D., Huang, J., Wielicki, B., Minnis, P., Weimer, C., Trepte, C., and Kuehn, R.: Global statistics of liquid water content and effective number concentration of water clouds over ocean derived from combined CALIPSO and MODIS measurements, Atmos. Chem. Phys., 7, 3353-3359, https://doi.org/10.5194/acp-7-3353-2007, 2007.

Hu, Y. X. and Stamnes, K.: An Accurate Parameterization of the Radiative Properties of Water Clouds Suitable for Use in Climate Models, J. Climate, 6, 728-742, https://doi.org/10.1175/15200442(1993)006<0728:AAPOTR>2.0.CO;2, 1993.

Huang, S.-S.: On the Eddington Approximation, Astrophys. J., 152, 841, https://doi.org/10.1086/149600, 1968. 
Joseph, J. H., Wiscombe, W. J., and Weinman, J. A.: The Delta-Eddington Approximation for Radiative Flux Transfer, J. Atmos. Sci., 33, 2452-2459, https://doi.org/10.1175/15200469(1976)033<2452:TDEAFR>2.0.CO;2, 1976.

Key, J.: Streamer Version 3.0, User's Guide, 107 pp., 2002.

Khairoutdinov, M., Randall, D., and DeMott, C.: Simulations of the Atmospheric General Circulation Using a Cloud-Resolving Model as a Superparameterization of Physical Processes, J. Atmos. Sci., 62, 2136-2154, https://doi.org/10.1175/JAS3453.1, 2005.

Koch, D. and Del Genio, A. D.: Black carbon semi-direct effects on cloud cover: review and synthesis, Atmos. Chem. Phys., 10, 7685-7696, https://doi.org/10.5194/acp-10-7685-2010, 2010.

Kopp, G. and Lean, J. L.: A new, lower value of total solar irradiance: Evidence and climate significance, Geophys. Res. Lett., 38, 1944-8007, https://doi.org/10.1029/2010GL045777, 2011.

Krieger, U. K., Mössinger, J. C., Luo, B., Weers, U., and Peter, T.: Measurement of the refractive indices of $\mathrm{H}_{2} \mathrm{SO}_{4}-\mathrm{HNO}_{3}-\mathrm{H}_{2} \mathrm{O}$ solutions to stratospheric temperatures, Appl. Optics, 39, 36913703, https://doi.org/10.1364/AO.39.003691, 2000.

Kurucz, R.: Synthetic infrared spectra, in Infrared Solar Physics, IAU Symp. edited by: Rabin, D. M. and Jefferies, J. T., Kluwer, Acad., Norwell, MA, 154, 1992.

Lacis, A. A. and Oinas, V.: A description of the correlated k distribution method for modeling nongray gaseous absorption, thermal emission, and multiple scattering in vertically inhomogeneous atmospheres, J. Geophys. Res.-Atmos., 96, 9027-9063, https://doi.org/10.1029/90JD01945, 1991.

Li, J. and Ramaswamy, V.: Four-Stream Spherical Harmonic Expansion Approximation for Solar Radiative Transfer, J. Atmos. Sci., 53, 1174-1186, https://doi.org/10.1175/15200469(1996)053<1174:FSSHEA>2.0.CO;2, 1996.

Li, J., Barker, H., Yang, P., and Yi, B.: On the aerosol and cloud phase function expansion moments for radiative transfer simulations, J. Geophys. Res.-Atmos., 120, 12128-12142, https://doi.org/10.1002/2015JD023632, 2015.

Liousse, C., Penner, J. E., Chuang, C., Walton, J. J., Eddleman, H., and Cachier, H.: A global three-dimensional model study of carbona- ceous aerosols, J. Geophys. Res.-Atmos., 101, 1941119432, https://doi.org/10.1029/95JD03426, 1996.

Lund-Myhre, C. E., Christensen, D. H., Nicolaisen, F. M., and Nielsen, C. J.: Spectroscopic Study of Aqueous $\mathrm{H}_{2} \mathrm{SO}_{4}$ at Different Temperatures and Compositions: Variations in Dissociation and Optical Properties, J. Phys. Chem. A, 107, 1979-1991, 2003.

Marsh, D. R., Mills, M.J. Kinnison, D.E. Lamarque, J.-F. Calvo, N., and Polvani, L. M.: Climate change from 1850 to 2005 simulated in CESM1(WACCM), J. Climate, 26, 7372-7391, https://doi.org/10.1175/JCLI-D-12-00558.1, 2013.

Martin, R. V., Jacob, D. J., Yantosca, R. M., Chin, M., and Ginoux, P.: Global and regional decreases in tropospheric oxidants from photochemical effects of aerosols, J. Geophys. Res. Atmos., 108, 4097, https://doi.org/10.1029/2002JD002622, 2003.

McGouldrick, K., Toon, O. B., and Grinspoon, D. H.: Sulfuric acid aerosols in the atmospheres of the terrestrial planets, Planet. Space Sc.i, 59, 934-941, https://doi.org/10.1016/j.pss.2010.05.020, 2011.

Meier, R. R., Anderson, G. P., Cantrell, C. A., Hall, L. A., Lean, J., Minschwaner, K., Shetter, R. E., Shettle, E. P., and Stamnes,
K.: Actinic radiation in the terrestrial atmosphere, J. Atmos. SolTerr. Phys., 59, 2111-2157, 1992.

Miles, N. L., Verlinde, J., and Clothiaux, E. E.: Cloud Droplet Size Distributions in Low-Level Stratiform Clouds, J. Atmos. Sci., 57, 295-311, https://doi.org/10.1175/15200469(2000)057<0295:CDSDIL>2.0.CO;2, 2000.

Mishchenko, M. I., Rossow, W. B., Macke, A., and Lacis, A. A.: Sensitivity of cirrus cloud albedo, bidirectional reflectance and optical thickness retrieval accuracy to ice particle shape, J. Geophys. Res.-Atmos., 101, 16973-16985, https://doi.org/10.1029/96JD01155, 1996.

Mishchenko, M. I., Videen, G., Babenko, V. A., Khlebtsov, N. G., and Wriedt, T.: T-matrix theory of electromagnetic scattering by particles and its applications: a comprehensive reference database, J. Quant. Spectrosc. Ra., 88, 357-406, 2004.

Mishchenko, M. I., Zakharova, N. T., Khlebtsov, N. G., Videen, G., and Wriedt, T.: Comprehensive thematic T-matrix ref- erence database: A 2014-2015 update, J. Quant. Spectrosc. Ra., 178, 276-283, https://doi.org/10.1016/j.jqsrt.2015.11.005, 2016.

Mlawer, E. J., Taubman, S. J., Brown, P. D., Iacono, M. J., and Clough, S. A.: Radiative transfer for inhomogeneous atmospheres: RRTM, a validated correlated-k model for the longwave, J. Geophys. Res.-Atmos., 102, 16663-16682, https://doi.org/10.1029/97JD00237, 1997.

Myhre, C. E. L., Christensen, D. H., Nicolaisen, F. M., and Nielsen, C. J.: Spectroscopic Study of Aqueous $\mathrm{H}_{2} \mathrm{SO}_{4}$ at Different Temperatures and Compositions: Variations in Dissociation and Optical Properties, J. Phys. Chem. A, 107, 1979-1991, https://doi.org/10.1021/jp026576n, 2003.

Myhre, G., Shindell, D., Bréon, F.-M., Collins, W., Fuglestvedt, J., Huang, J., Koch, D., Lamarque, J.-F., Lee, D., Mendoza, B., Nakajima, T., Robock, A., Stephens, G., Takemura, T., and Zhang, H.: Anthropogenic and Natural Radiative Forcing, in: Climate Change 2013: The Physical Science Basis, IPCC WGI Contribution to the Fifth Assessment Report, edited by: Stocker, T. F., Qin, D., Plattner, G.-K., Tignor, M., Allen, S. K., Boschung, J., Nauels, A., Xia, Y., Bex, V., and Midgley, P. M., Cambridge University Press Cambridge, United Kingdom, 659-740, 2013.

Neu, J. L., Prather, M. J., and Penner, J. E.: Global atmospheric chemistry: Integrating over fractional cloud cover, J. Geophys. Res.-Atmos., 112, D11306, https://doi.org/10.1029/2006jd008007, 2007.

Painemal, D., Greenwald, T., Cadeddu, M., and Minnis, P.: First extended validation of satellite microwave liquid water path with ship-based observations of marine low clouds, Geophys. Res. Lett., 43, 6563-6570, https://doi.org/10.1002/2016GL069061, 2016.

Palancar, G. G., Shetter, R. E., Hall, S. R., Toselli, B. M., and Madronich, S.: Ultraviolet actinic flux in clear and cloudy atmospheres: model calculations and aircraftbased measurements, Atmos. Chem. Phys., 11, 5457-5469, https://doi.org/10.5194/acp-11-5457-2011, 2011.

Palmer, K. F. and Williams, D.: Optical properties of water in the near infrared, J. Opt. Soc. Am., 64, 1107-1110, https://doi.org/10.1364/JOSA.64.001107, 1974.

Pincus, R., Barker, H. W., and Morcrette, J. J.: A fast, flexible, approximate technique for computing radiative transfer in inhomogeneous cloud fields, J. Geophys. Res.-Atmos., 108, D4376, https://doi.org/10.1029/2002jd003322, 2003. 
Prather, M. J.: Photolysis rates in correlated overlapping cloud fields: Cloud-J 7.3c, Geosci. Model Dev., 8, 2587-2595, https://doi.org/10.5194/gmd-8-2587-2015, 2015.

Rezaei, S., Hernandez-Calderon, C. A., Mirzamohammadi, S., Bozorgzadeh, E., Veidenbaum, A. V., Nicolau, A., and Prather, M. J.: Data-rate-aware FPGA-based Acceleration Framework for Streaming Applications, ReConFig, 2016.

Rozanov, V., Rozanov, A., Kokhanovsky, A., and Burrows, J.: Radiative transfer through terrestrial atmosphere and ocean: Software package SCIATRAN, J. Quant. Spectrosc. Ra., 133, 13-71, https://doi.org/10.1016/j.jqsrt.2013.07.004, 2014.

Sander, S., Friedl, R. R., Abbatt, J. P. D., Barker, J. R., Burkholder, J. B., Golden, D. M., Kolb, C. E., Kurylo, M. J., Moortgat, G. K., Wine, P. H., and Orkin, R. E. H. V. L.: Chemical Kinetics and Photochemical Data for Use in Atmospheric Studies, NASA Panel for Data Evaluation, 2011.

Søvde, O. A., Prather, M. J., Isaksen, I. S. A., Berntsen, T. K., Stordal, F., Zhu, X., Holmes, C. D., and Hsu, J.: The chemical transport model Oslo CTM3, Geosci. Model Dev., 5, 1441-1469, https://doi.org/10.5194/gmd-5-1441-2012, 2012.

Stamnes, K., Tsay, S. C., Wiscombe, W., and Jayaweera, K.: Numerically Stable Algorithm for Discrete-Ordinate-Method Radiative-Transfer in Multiple-Scattering and Emitting Layered Media, Appl. Optics, 27, 2502-2509, 1988.

Sukhodolov, T., Rozanov, E., Ball, W. T., Bais, A., Tourpali, K., Shapiro, A. I., Telford, P., Smyshlyaev, S., Fomin, B., Sander, R., Bossay, S., Bekki, S., Marchand, M., Chipperfield, M. P., Dhomse, S., Haigh, J. D., Peter, T., and Schmutz, W.: Evaluation of simulated photolysis rates and their response to solar irradiance variability, J. Geophys. Res.-Atmos., 121, 6066-6084, https://doi.org/10.1002/2015JD024277, 2016.

Telford, P. J., Abraham, N. L., Archibald, A. T., Braesicke, P., Dalvi, M., Morgenstern, O., O'Connor, F. M., Richards, N. A. D., and Pyle, J. A.: Implementation of the Fast-JX Photolysis scheme (v6.4) into the UKCA component of the MetUM chemistry-climate model (v7.3), Geosci. Model Dev., 6, 161177, https://doi.org/10.5194/gmd-6-161-2013, 2013.
Tie, X., Madronich, S., Walters, S., Zhang, R., Rasch, P., and Collins, W.: Effect of clouds on photolysis and oxidants in the troposphere, J. Geophys. Res.-Atmos., 108, 4642, https://doi.org/10.1029/2003JD003659, 2003.

Tilmes, S., Mills, M. J., Niemeier, U., Schmidt, H., Robock, A., Kravitz, B., Lamarque, J.-F., Pitari, G., and English, J. M.: A new Geoengineering Model Intercomparison Project (GeoMIP) experiment designed for climate and chemistry models, Geosci. Model Dev., 8, 43-49, https://doi.org/10.5194/gmd-8-43-2015, 2015.

Warren, S. G.: Optical constants of ice from the ultraviolet to the microwave, Appl. Optics, 23, 1206-1225, https://doi.org/10.1364/AO.23.001206, 1984.

Wild, O., Zhu, X., and Prather, M. J.: Fast-J: Accurate Simulation of In- and Below-Cloud Photolysis in Tropospheric Chemical Models, J. Atmos. Chem., 37, 245-282, https://doi.org/10.1023/A:1006415919030, 2000.

Wiscombe, W. J.: The Delta-M Method: Rapid Yet Accurate Radiative Flux Calculations for Strongly Asymmetric Phase Functions, J. Atmos. Sci., 34, 1408-1422, https://doi.org/10.1175/15200469(1977)034<1408:TDMRYA>2.0.CO;2, 1977.

Yang, P., Liou, K.-N., Bi, L., Liu, C., Yi, B., and Baum, B. A.: On the Radiative Properties of Ice Clouds: Light Scattering, Remote Sensing, and Radiation Parameterization, Adv. Atmos. Sci., 32, 32-63, https://doi.org/10.1007/s00376-014-0011-z, 2015.

Zhou, C. and Yang, P.: Backscattering peak of ice cloud particles, Opt. Express, 23, 11995-12003, https://doi.org/10.1364/OE.23.011995, 2015. 